\title{
IDENTIFICATION AND CONTROL OF SALTWATER INTRUSION BY ADR APPROACH IN THE COASTAL AQUIFERS OF TUTICORIN, INDIA
}

\author{
Glory Selvamano, J. ${ }^{1 *}-$ PrinCE ARUl RAJ, G. ${ }^{2}-$ JeYANTHI, J. ${ }^{3}$ \\ ${ }^{I}$ Sardar Raja College of Engineering, Alangulam-627808, Tamilnadu, India \\ ${ }^{2}$ Karunya Institute of Technology and Sciences, Coimbatore-641114, Tamilnadu, India \\ ${ }^{3}$ Government College of Technology, Coimbatore-641013, Tamilnadu, India \\ *Corresponding author \\ e-mail: gloryjason2007@gmail.com \\ (Received $13^{\text {th }}$ Nov 2018; accepted $16^{\text {th }}$ Jan 2019)
}

\begin{abstract}
Due to industrial growth and urbanisation, excessive usage of groundwater resulted in a problem of saltwater intrusion in Tuticorin, India where control and management is very much essential. In the present study, groundwater samples are collected and analysed from 38 observation wells in years 2014 and 2018. Thirteen parameters namely pH, Electrical Conductivity (EC), Total Dissolved Solids (TDS), Total Hardness (TH), Calcium $\left(\mathrm{Ca}^{2+}\right)$, Magnesium $\left(\mathrm{Mg}^{2+}\right)$, Sodium $\left(\mathrm{Na}^{+}\right)$Potassium $\left(\mathrm{K}^{+}\right)$, Bicarbonate $\left(\mathrm{HCO}_{3}^{-}\right)$, Chloride $\left(\mathrm{Cl}^{-}\right)$, Sulphate $\left(\mathrm{SO}_{4}{ }^{2-}\right)$ Nitrate $\left(\mathrm{NO}_{3}^{-}\right)$and Fluoride $\left(\mathrm{F}^{-}\right)$are determined and considered in calculating the Water Quality Index (WQI) based upon weighted arithmetic index method. Geographical information system (GIS) is used to interpolate water quality data by inverse distance weighted method. Experimental investigation indicates saltwater intrusion in the coastal aquifer of Tuticorin is due to excessive withdrawal of groundwater. Potential intrusion of saltwater is studied with respect to distance of observation wells from seashore. Finite Element Modelling of Flow (FEFLOW) is used to select the optimum pumping and recharge rate to control saltwater intrusion. A model is calibrated with hydraulic head measured using piezometer in the observation well, as well as salt concentration. The model is simulated using three different groundwater scenarios such as Abstraction, Recharge and combined Abstraction, Desalination Recharge (ADR) method. The simulation results depicted that the planned ADR system accomplishes significantly better than using abstraction or recharge well.
\end{abstract}

Keywords: GIS, groundwater management, FEFLOW, hydraulic head, water quality index

\section{Introduction}

Groundwater extraction is required in several coastal areas where the freshwater supply from surface sources is not sufficient. However, extreme groundwater abstraction may lead to intrusion of saltwater into the aquifer, henceforth salinity increases making the groundwater unfit for human intake (Rao et al., 2004). Saltwater contamination arises due to vertical movement across interconnected aquifers through open well or bore well (Barlow and Reichard, 2010). Jebastina and Prince Arulraj (2016) analysed groundwater and determined the quality changes due to silicate weathering and dissolution of carbonate. Assessment of groundwater quality plays a vital role in our society, predominantly concerning health (Sener et al., 2009). One of the important and easy methods for evaluating intrusion of saltwater in coastal areas is periodic analysis of groundwater chemistry (Todd, 1980). The information of hydrochemistry is significant to assess the groundwater quality in Coimbatore in which the groundwater is used for drinking, agriculture, and industrial purposes (Jebastina and Prince Arulraj, 2017). In recent years water quality index (WQI) has become an 
essential method to assess the quality of groundwater. Tavassoli and Mohammadi (2017) assessed the vulnerability of groundwater based on WQI values. WQI method handles complex data of water quality parameter and provides simplified output to understand water quality problem. Idowu et al. (2016) used geographical information system (GIS) as a prevailing tool to assess vulnerability of seawater intrusion and effective management of Groundwater.

Todd (1974) discussed various methods of preventing saltwater from polluting groundwater sources including; decrease of pumping rates, repositioning of extraction wells, use of sub-surface walls, recharge of water by natural and artificial methods, abstraction of saline groundwater and any combination of above techniques. El Mokhtar et al. (2018) developed model for planning and management of sustainable use of groundwater resources in the coastal aquifer of Fum Al Wad, Morrocco. Groundwater model evidenced that pumping of saline water from coastal aquifers would mitigate the rate at which saltwater flows into the aquifer so that quality of water improved. Hussain et al. (2015) suggested continuous Abstraction of brackish water, desalination and recharge techniques control saltwater intrusion effectively in coastal aquifers. Thomas et al. (2016) used simulation model to determine proper pumping strategy for the management of Coastal aquifers. Finite Element Modelling of Flow (FEFLOW) is a software used for groundwater modelling capable of simulating flow.

The main objective of this study is to evaluate the quality of groundwater using WQI in Tuticorin, India and thematically denote it using Geographical information system (GIS) for the better understanding of the current scenario at a glance. An effort has been made in the coastal aquifers of Tuticorin to protect the wells from seawater intrusion by FEFLOW code, using hydrogeological and hydro chemical data.

\section{Location of the study area}

The study area Tuticorin is situated in the southeast shoreline of Tamil Nadu, India and is positioned between $8^{\circ} 19^{\prime}$ to $9^{\circ} 22^{\prime} \mathrm{N}$ latitude and $77^{\circ} 40^{\prime}$ to $78^{\circ} 23^{\prime} \mathrm{E}$ longitude. The area is bound by the Gulf of Mannar in the east and Tirunelveli district, Tamil Nadu in the west. The main occupation of the people is agriculture. Many chemical industries, food processing industries, salt industries are situated in Tuticorin. Many industries are established after the construction of the port and Tuticorin became a district in the year 1986. After the formation, the economic development has been boosted and began to develop rapidly. Therefore, the urban expansion acquired in various areas of the district. The slope of the terrain is mild in the western part and flat in the eastern part. The location map of the study area is shown in Figure. 1.

\section{Methodology}

\section{Sample collection}

A total of 38 groundwater samples are collected from open and bore wells. The location of observation wells is shown in Figure 2. Each sample is collected in $1000 \mathrm{~mL}$ acid-washed polyethylene HDPE bottle. The bottle is fully filled with water such that no air bubble is stuck within the water sample. To avoid evaporation, the bottles are closed with double plastic caps. Precaution is also taken to prevent agitation of sample during transfer from field to the lab. 


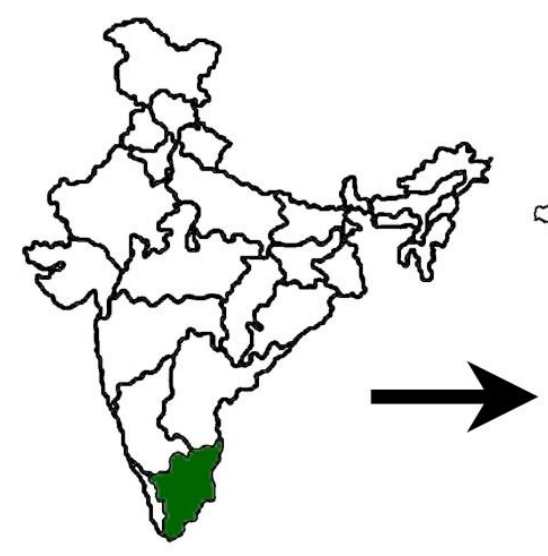

India

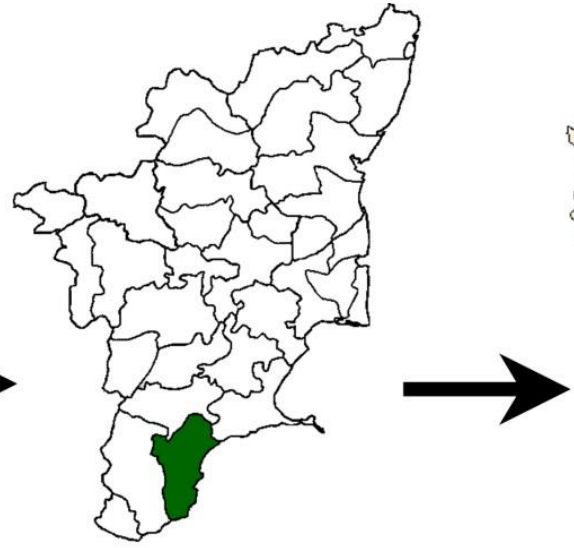

Tamil Nadu

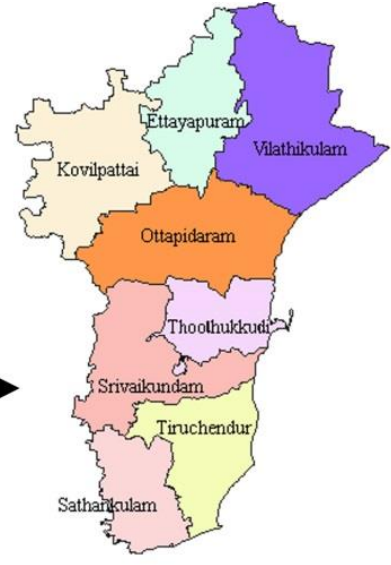

Tuticorin

Figure 1. Location map of study area

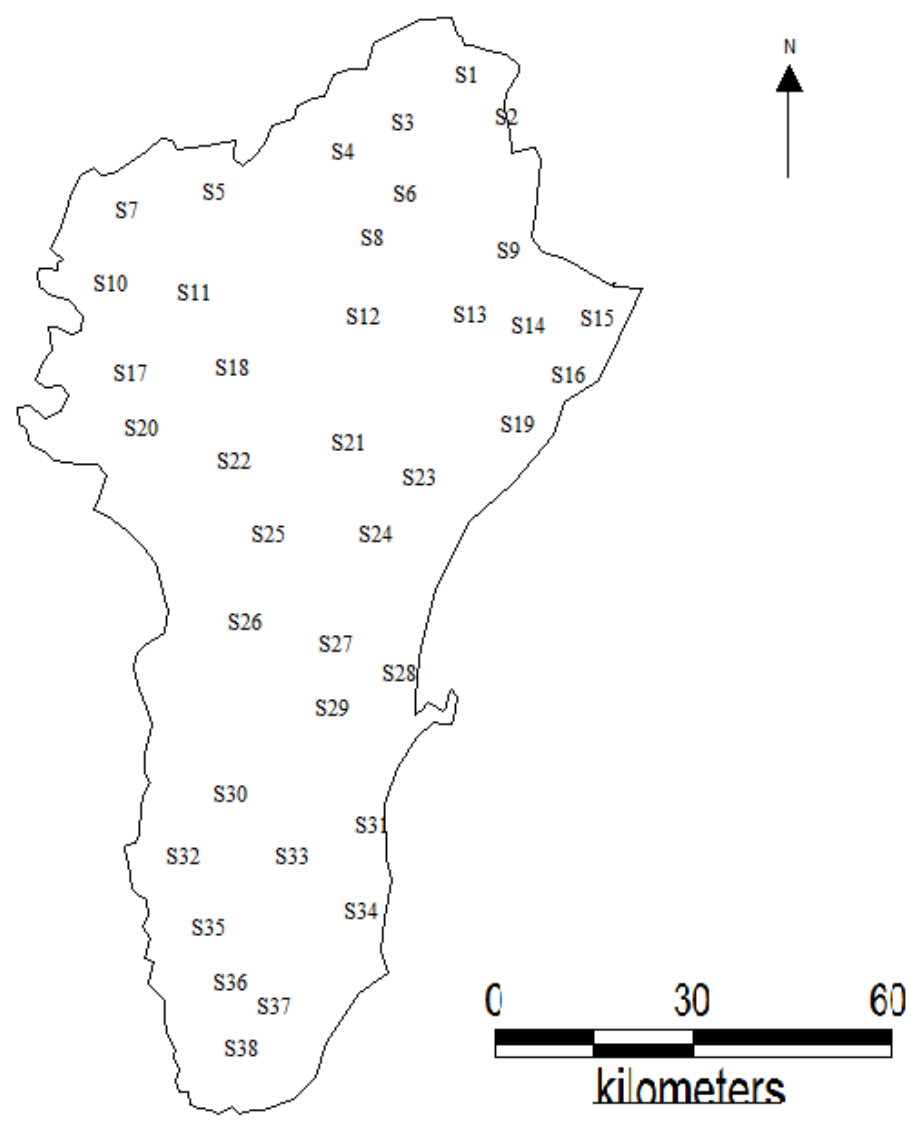

Figure 2. Location of observation wells

\section{Laboratory measurements}

The physico-chemical analysis was conducted using following methods and equipment as shown in Table 1. 
Table 1. Method and instrument used in water quality analysis

\begin{tabular}{c|c|c|c}
\hline S.No & Parameter & Method & Instrument/equipment \\
\hline 1. & $\mathrm{pH}$ & Electrometric & pH meter \\
2. & EC (electrical conductivity) & Electrometric & Conductivity meter \\
3. & TDS (total dissolved solids) & Electrometric & Conductivity meter \\
4. & $\mathrm{TH}$ (total hardness) & Titration by EDTA & - \\
5. & $\mathrm{Ca}^{2+}$ (Calcium) & Titration by EDTA & - \\
6. & $\mathrm{Mg}^{2+}$ (Magnesium) & Titration by EDTA & Flame photometer \\
7. & $\mathrm{Na}^{+}$(Sodium) & Flame emission & Flame photometer \\
8. & $\mathrm{~K}^{+}$(Potassium) & Flame emission & - \\
9. & $\mathrm{HCO}_{3}^{-}$(Bicarbonate) & Titration by $\mathrm{H}_{2} \mathrm{SO}_{4}$ & - \\
10. & $\mathrm{Cl}^{-}$(Chlorine) & Titration by AgNO & - \\
11. & $\mathrm{SO}_{4}^{2-}$ (Sulphate) & Use wavelength of $420 \mathrm{~nm}$ & UV-VIS spectrophotometer \\
12. & $\mathrm{NO}_{3}{ }^{-}$(Nitrate) & Use wavelength of $220 \mathrm{~nm}$ & UV-VIS spectrophotometer \\
13. & $\mathrm{~F}^{-}$(Fluoride) & Ion-selective electrode $($ISE) & pH meter \\
\hline
\end{tabular}

All concentrations are denoted in milligrams per litre $(\mathrm{mg} / \mathrm{L})$, excluding the $\mathrm{pH}$ and EC

\section{Determination of water quality index (WQI)}

To calculate WQI, thirteen parameters namely $\mathrm{pH}, \mathrm{EC}$, TDS, TH, $\mathrm{Ca}^{2+}, \mathrm{Mg}^{2+}, \mathrm{Na}^{+}$ $\mathrm{K}^{+}, \mathrm{HCO}_{3}{ }^{-}, \mathrm{Cl}^{-}, \mathrm{SO}_{4}{ }^{2-} \mathrm{NO}_{3}{ }^{-}$and $\mathrm{F}^{-}$are considered into account. The following few steps are followed in calculating WQI.

\section{Parameter selection}

The selection of parameters depends on several aspects, such as the purpose of the index, the importance of the parameter, and the availability of data (Stigter et al., 2006). For the assessment of drinking water quality, priority should be given to those parameters which are known to be important to health and to be present in significant concentrations in the water source (Ramesh et al., 2010). Table 2 gives the details of the weights and relative weights assigned to various parameters.

Table 2. Weights for various parameters

\begin{tabular}{c|c|c|c|c|c}
\hline \multirow{2}{*}{$\begin{array}{c}\text { Water quality } \\
\text { parameter }\end{array}$} & Units & \multicolumn{2}{|c|}{ WHO (2017) } & Weight & $\begin{array}{c}\text { Relative weight } \\
\left(\mathbf{W}_{\mathbf{R}}\right)\end{array}$ \\
\cline { 3 - 4 } & $\begin{array}{c}\text { Most desirable } \\
\text { limit }\end{array}$ & $\begin{array}{c}\text { Maximum allowable } \\
\text { limit }\end{array}$ & \\
\hline $\mathrm{pH}$ & - & 6.5 & 8.5 & 4 & 0.09 \\
$\mathrm{EC}$ & $\mu \mathrm{s} / \mathrm{cm}$ & 780 & 3125 & - & - \\
$\mathrm{HCO}_{3}$ & $\mathrm{mg} / \mathrm{L}$ & - & 300 & 1 & 0.03 \\
$\mathrm{TDS}$ & $\mathrm{mg} / \mathrm{L}$ & 500 & 1500 & 5 & 0.11 \\
$\mathrm{~F}$ & $\mathrm{mg} / \mathrm{L}$ & - & 1.5 & 5 & 0.11 \\
$\mathrm{Cl}$ & $\mathrm{mg} / \mathrm{L}$ & 200 & 600 & 5 & 0.11 \\
$\mathrm{NO}_{3}$ & $\mathrm{mg} / \mathrm{L}$ & 45 & - & 5 & 0.11 \\
$\mathrm{SO}_{4}$ & $\mathrm{mg} / \mathrm{L}$ & 200 & 400 & 5 & 0.11 \\
$\mathrm{Na}$ & $\mathrm{mg} / \mathrm{L}$ & - & 200 & 4 & 0.09 \\
$\mathrm{Ca}$ & $\mathrm{mg} / \mathrm{L}$ & 75 & 200 & 3 & 0.07 \\
$\mathrm{Mg}$ & $\mathrm{mg} / \mathrm{L}$ & 30 & 150 & 3 & 0.07 \\
$\mathrm{~K}$ & $\mathrm{mg} / \mathrm{L}$ & - & 10 & 2 & 0.05 \\
$\mathrm{TH}$ & $\mathrm{mg} / \mathrm{L}$ & 300 & 600 & 2 & 0.05 \\
& & & & $\sum \mathrm{W}=44$ & $\sum \mathrm{W}_{\mathrm{R}}=1$ \\
\hline
\end{tabular}

Drinking water quality standards given by the World Health Organization (WHO, 2017) 


\section{Weight assignment}

The assignment of weight to water quality parameters is to represent the importance of each parameter in the overall water quality. Higher weightage implies greater importance of the variable with respect to public health (Song and Kim, 2009). Therefore, each of the selected parameters has been given a weight (W) 1 to 5 , depending on their importance. The chosen weights are tabulated in Table 2. These weights are assumed based on the data available from previous studies (Serrekawo and Karoppannan, 2018; Ramakrishnaiah et al., 2009; Gebrehiwot et al., 2011; Kalpana et al., 2014).

\section{Relative weight calculation}

Relative weight $\left(\mathrm{W}_{\mathrm{R}}\right)$ can be determined by dividing the individual weight of each parameter $\left(\mathrm{W}_{\mathrm{I}}\right)$ by the sum of weight of all selected parameters $(W)$ shown in Equation 1.

$$
W_{R}=\frac{W_{I}}{W}
$$

where $\mathrm{W}_{\mathrm{R}}$ is the relative weight, $\mathrm{W}_{\mathrm{I}}$ is the weight of the parameter under consideration.

\section{Quality rating calculation}

The fourth step is the calculation of quality rating $\left(\mathrm{Q}_{\mathrm{I}}\right)$ for each parameter, is given in Equation 2.

$$
Q_{I}=\frac{C_{I}}{S_{I}} \times 100
$$

where $\mathrm{Q}_{\mathrm{I}}$ is quality rating, $\mathrm{C}_{\mathrm{I}}(\mathrm{mg} / \mathrm{L})$ is the concentration of each parameter in water sample, and $S_{I}(m g / L)$ is the Bureau of Indian standard for chemical parameter.

\section{Sub-index calculation}

The sub-index for each chemical parameter is determined using Equation 3.

$$
S_{I}=W_{R} \times Q_{I}
$$

$\mathrm{S}_{\mathrm{I}}$ is the sub-index of the $i$ th parameter. It combines its quality rating as well as its assigned weight.

\section{Calculation of water quality index}

The overall WQI is calculated by summation of all sub index values of each groundwater sample as follows (Eq. 4):

$$
W Q I=\sum S_{I}
$$




\section{Classification of water quality index}

Higher WQI values indicate worse water quality, and lower values indicate excellent water quality. The detail description of WQI range and its classification is given in Table 3.

Table 3. Classification of WQI values

\begin{tabular}{c|c}
\hline WQI range & Category of water \\
\hline$<50$ & Excellent \\
$50-100$ & Good \\
$100-200$ & Poor \\
$200-300$ & Very poor \\
$>300$ & Unfit for drinking purpose \\
\hline
\end{tabular}

\section{GIS analysis}

GIS is an effective method for spatial analysis and integration of the data to derive needed outputs and modelling. GIS can be beneficial for taking fast conclusions as graphical illustration would make visualization easy for policy makers. The study area of Tuticorin district is digitized from the survey of India toposheet using QGIS. The exact locations of observation wells are determined in the field using GPS and the precise longitudes and latitudes of observation wells are imported in GIS platform. Inverse Distance Weighted (IDW) interpolation method was used for spatial modelling of WQI and the parameter values are classified according to Table 3.

\section{Model development (FEFLOW)}

The first step in FEM analysis is the generation of mesh that fills the envisioned model domain with elements as shown in Figure 3. Based on the finite-element mesh, initial hydraulic head, boundary conditions and material properties are defined as given in Table 4.
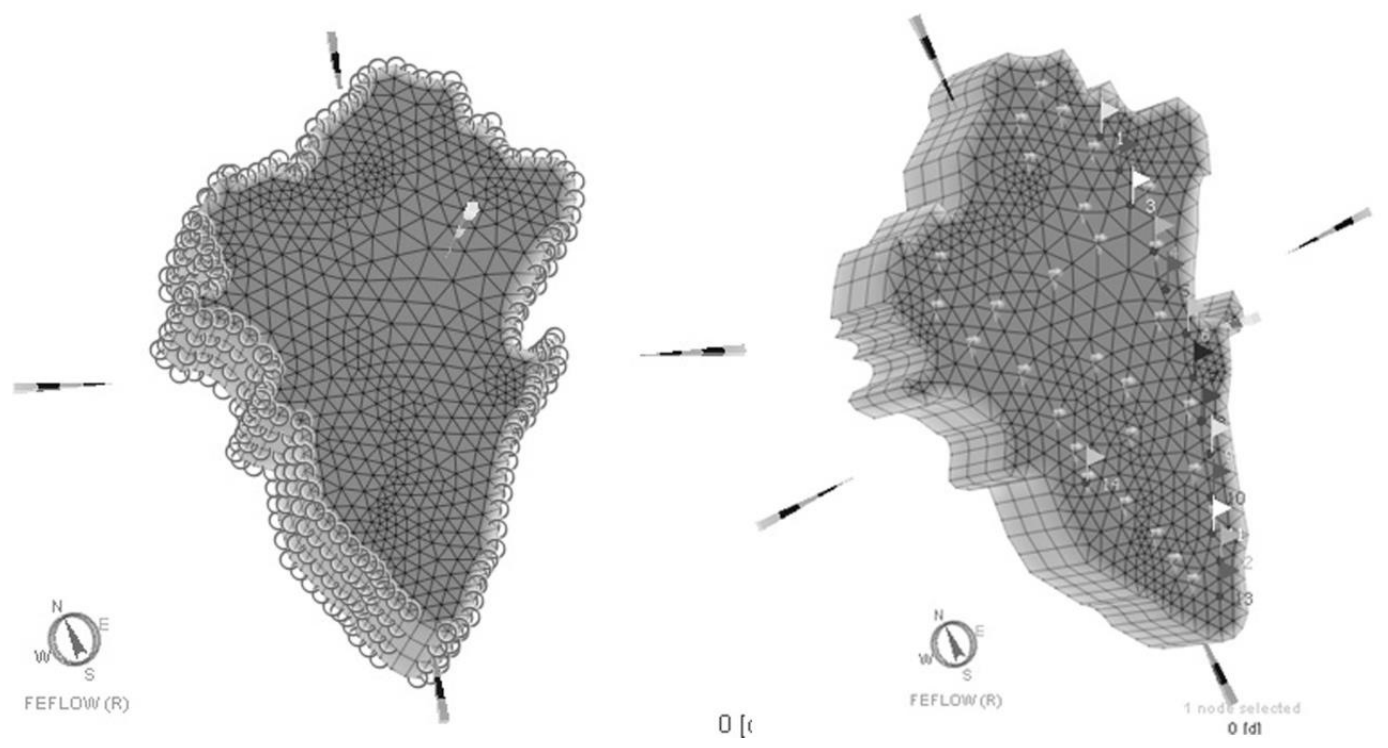

Figure 3. Generation of finite element mesh 
Table 4. Data used for model development

\begin{tabular}{|c|c|}
\hline Parameter & Value \\
\hline \multicolumn{2}{|l|}{ Physical parameters } \\
\hline \multicolumn{2}{|l|}{ Mesh } \\
\hline Element type & Triangle prism \\
\hline Mesh element & 3351 \\
\hline Mesh node & 2496 \\
\hline Number of layer & 1 \\
\hline Number of slice & 2 \\
\hline Total model boundary areas & $4621 \mathrm{~km}^{2}$ \\
\hline \multicolumn{2}{|l|}{ Materials properties and boundary conditions (BC) } \\
\hline Hydraulic conductivity $\mathrm{K}_{\mathrm{X}}, \mathrm{K}_{\mathrm{Y}}, \mathrm{K}_{\mathrm{Z}}$ & $4.5 \times 10^{-4} \mathrm{~m} / \mathrm{s}, 4.5 \times 10^{-4} \mathrm{~m} / \mathrm{s}, 1.5 \times 10^{-4} \mathrm{~m} / \mathrm{s}$ \\
\hline Specific storage (fluid flux) & .002 \\
\hline Total porosity & .35 \\
\hline Longitude dispersivity & 1 \\
\hline Transverse dispersivity & $0.1 \mathrm{~m}$ \\
\hline Density ratio & $1025 \mathrm{~kg} / \mathrm{m}^{3}$ \\
\hline Constant head & $0 \mathrm{~m}$ \\
\hline Recharge concentration & $0 \mathrm{mg} / \mathrm{L}$ \\
\hline Constant head concentration & $35000 \mathrm{mg} / \mathrm{L}$ \\
\hline Initial concentration & 20.22 \\
\hline Initial head & $1.011 \mathrm{~m}$ \\
\hline
\end{tabular}

The model is calibrated by changing the aquifer properties and the boundary conditions in order to obtain a model appropriate to simulate the groundwater flow and the mass concentration distribution, within the calibration target. In the calibration process, a trial and error technique is carried out in order to reduce the variance between the simulated and the measured values. Once the model is calibrated; it is run to verify various scenarios as shown in Table 5 where the extrapolative simulations are performed to minimize saltwater intrusion in the unconfined aquifer.

Table 5. Three scenarios used for model simulation

\begin{tabular}{c|c}
\hline Simulation & Description \\
\hline Scenario A & Abstraction of saline water $1000 \mathrm{~m}^{3} / \mathrm{day}$ \\
Scenario B & $\begin{array}{c}\text { Recharge of treated water at the rate of } 500 \mathrm{~m}^{3} / \text { day } \\
\text { Scenario C }\end{array}$ \\
& $\begin{array}{c}\text { Abstraction of saline water } 1000 \mathrm{~m}^{3} / \text { day, desalination and recharge of treated } \\
\text { water at the rate of } 500 \mathrm{~m}^{3} / \text { day }\end{array}$ \\
\hline
\end{tabular}

\section{Results}

\section{Laboratory measurements}

The water quality characteristics of the groundwater samples during the year 2014 and 2018 are determined and the values are given in Tables 6 and 7. 
Table 6. Physico-chemical values in 2014

\begin{tabular}{|c|c|c|c|c|c|c|c|c|c|c|c|c|c|c|c|c|}
\hline $\begin{array}{l}\text { Well } \\
\text { No }\end{array}$ & Latitude & ongitude & pH & EC & TDS & $\mathbf{T H}$ & $\mathbf{C a}$ & $\mathbf{K}$ & $\mathbf{N a}$ & Mg & $\mathrm{HCO}_{3}$ & Cl & $\mathrm{SO}_{4}$ & $\mathbf{F}$ & $\mathrm{NO}_{3}$ & $\begin{array}{l}\text { WQI } \\
2014\end{array}$ \\
\hline S1 & $09^{\circ} 13^{\prime} 47^{\prime \prime}$ & $78^{\circ} 08^{\prime} 12^{\prime \prime}$ & 7.5 & 1360 & 763 & 370 & 54 & 9 & 145 & 57 & 342 & 220 & 33 & 0.38 & 17 & 130 \\
\hline S2 & $09^{\circ} 14^{\prime} 09^{\prime \prime}$ & $78^{\circ} 01^{\prime} 50^{\prime \prime}$ & 8 & 1440 & 823 & 420 & 60 & 4 & 145 & 66 & 354 & 149 & 154 & 0.38 & 15 & 121 \\
\hline S3 & $09^{\circ} 17^{\prime} 47^{\prime \prime}$ & $78^{\circ} 03^{\prime} 56^{\prime \prime}$ & 8.5 & 1190 & 749 & 305 & 84 & 3 & 124 & 23 & 73 & 21 & 442 & 0.65 & 1 & 86 \\
\hline S4 & $9^{\circ} 10^{\prime} 45^{\prime \prime}$ & $77^{\circ} 52^{\prime} 25^{\prime \prime}$ & 8.3 & 770 & 458 & 235 & 48 & 7 & 69 & 28 & 142 & 99 & 65 & 0.36 & 12 & 77 \\
\hline S5 & $09^{\circ} 08^{\prime} 30^{\prime \prime}$ & $78^{\circ} 02^{\prime} 15^{\prime \prime}$ & 8 & 3070 & 1985 & 550 & 80 & 12 & 460 & 85 & 366 & 355 & 624 & 1.04 & 42 & 258 \\
\hline S6 & $09^{\circ} 08^{\prime} 57^{\prime \prime}$ & $77^{\circ} 42^{\prime} 10^{\prime \prime}$ & 8.8 & 2930 & 1612 & 540 & 108 & 2 & 414 & 66 & 641 & 482 & 192 & 1.14 & 2 & 254 \\
\hline S7 & $09^{\circ} 09^{\prime} 00^{\prime \prime}$ & 7759'54" & 7.8 & 1270 & 755 & 330 & 42 & 7 & 145 & 55 & 336 & 124 & 154 & 1.09 & 14 & 116 \\
\hline S8 & $09^{\circ} 07^{\prime} 45^{\prime \prime}$ & $78^{\circ} 10^{\prime} 07^{\prime \prime}$ & 8.2 & 3950 & 2290 & 770 & 148 & 5 & 552 & 97 & 647 & 709 & 384 & 1.57 & 16 & 362 \\
\hline S9 & $09^{\circ} 08^{\prime} 40^{\prime \prime}$ & $77^{\circ} 47^{\prime} 15^{\prime \prime}$ & 7.7 & 1410 & 1045 & 810 & 152 & 23 & 177 & 104 & 232 & 234 & 68 & 0.32 & 38 & 179 \\
\hline $\mathrm{S} 10$ & 09 & $77^{\circ} 48$ & 8.8 & 690 & 399 & 185 & 8 & 14 & 69 & 40 & 155 & 53 & 96 & 0.26 & 4 & 63 \\
\hline $\mathrm{S} 11$ & $09^{\circ} 06^{\prime} 00^{\prime \prime}$ & $78^{\circ} 00^{\prime} 40^{\prime \prime}$ & 7.7 & 3610 & 2257 & 820 & 296 & 12 & 460 & 19 & 311 & 837 & 154 & 0.15 & 73 & 375 \\
\hline $\mathrm{S} 12$ & $08^{\circ} 33^{\prime} 00^{\prime \prime}$ & $78^{\circ} 01^{\prime} 00^{\prime \prime}$ & 8.2 & 740 & 394 & 280 & 40 & 4 & 41 & 44 & 74 & 170 & 29 & 0.46 & 5 & 89 \\
\hline $\mathrm{S} 13$ & $09^{\circ} 04^{\prime} 00^{\prime \prime}$ & $78^{\circ} 10^{\prime} 35^{\prime \prime}$ & 8.5 & 1730 & 1028 & 400 & 60 & 2 & 225 & 61 & 354 & 191 & 288 & 0.78 & 3 & 145 \\
\hline S14 & 7" & $78^{\circ} 08^{\prime} 12^{\prime \prime}$ & 8.6 & 2600 & 1606 & 370 & 96 & 16 & 442 & 32 & 689 & 326 & 216 & 0.63 & 27 & 215 \\
\hline $\mathrm{S} 15$ & $09^{\circ} 00$ & $78^{\circ} 11^{\prime} 50^{\prime \prime}$ & 7.9 & 550 & 296 & 235 & 40 & 4 & 20 & 33 & 203 & 21 & 41 & 0.56 & 3 & 51 \\
\hline S16 & $09^{\circ} 03^{\prime} 35^{\prime \prime}$ & $77^{\circ} 44^{\prime} 50^{\prime \prime}$ & 8.6 & 1520 & 936 & 230 & 16 & 7 & 253 & 46 & 293 & 170 & 214 & 1.4 & 16 & 136 \\
\hline S17 & $08^{\circ} 59^{\prime} 40^{\prime \prime}$ & $77^{\circ} 51^{\prime} 38^{\prime \prime}$ & 7.9 & 730 & 425 & 285 & 50 & 9 & 35 & 39 & 109 & 117 & 27 & 0.29 & 18 & 81 \\
\hline S18 & $09^{\circ} 00^{\prime} 00^{\prime \prime}$ & $77^{\circ} 58^{\prime} 00^{\prime \prime}$ & 8 & 1610 & 966 & 240 & 20 & 1 & 276 & 46 & 421 & 206 & 144 & 1.02 & 14 & 39 \\
\hline S19 & $09^{\circ} 03^{\prime} 00^{\prime \prime}$ & $77^{\circ} 54^{\prime} 00^{\prime \prime}$ & 7.6 & 2330 & 1527 & 350 & 48 & 108 & 350 & 56 & 470 & 340 & 173 & 0.58 & 49 & 245 \\
\hline $\mathrm{S} 20$ & $09^{\circ} 01^{\prime} 10^{\prime \prime}$ & $78^{\circ} 02^{\prime} 45^{\prime \prime}$ & 8.4 & 2100 & 1376 & 260 & 40 & 88 & 331 & 39 & 439 & 234 & 264 & 1.48 & 35 & 212 \\
\hline S21 & $08^{\circ} 56^{\prime} 40^{\prime \prime}$ & $77^{\circ} 52^{\prime} 00^{\prime \prime}$ & 8.4 & 1740 & 1060 & 370 & 28 & 121 & 161 & 73 & 366 & 255 & 115 & 0.22 & 27 & 199 \\
\hline $\mathrm{S} 22$ & $08^{\circ} 54^{\prime} 45^{\prime \prime}$ & $78^{\circ} 01^{\prime} 25^{\prime \prime}$ & 7.6 & 3050 & 1752 & 1320 & 240 & 37 & 78 & 175 & 238 & 709 & 96 & 0.33 & 67 & 353 \\
\hline $\mathrm{S} 23$ & $08^{\circ} 52^{\prime} 30^{\prime \prime}$ & $78^{\circ} 02^{\prime} 15^{\prime \prime}$ & 7.5 & 2540 & 1598 & 540 & 100 & 66 & 304 & 70 & 329 & 404 & 178 & 0.1 & 70 & 252 \\
\hline $\mathrm{S} 24$ & $08^{\circ} 51^{\prime} 38^{\prime \prime}$ & $77^{\circ} 54^{\prime} 00^{\prime \prime}$ & 7.9 & 2530 & 1504 & 560 & 80 & 78 & 290 & 87 & 537 & 390 & 192 & 0.41 & 26 & 250 \\
\hline $\mathrm{S} 25$ & $08^{\circ} 47^{\prime} 25^{\prime \prime}$ & $77^{\circ} 52^{\prime} 15^{\prime \prime}$ & 8.6 & 590 & 315 & 200 & 42 & 20 & 32 & 23 & 212 & 32 & 25 & 0.17 & 2 & 55 \\
\hline S26 & $08^{\circ} 48^{\prime} 35^{\prime \prime}$ & $78^{\circ} 01^{\prime} 25^{\prime \prime}$ & 7.5 & 880 & 503 & 260 & 360 & 37 & 368 & 87 & 178 & 465 & 336 & 0.23 & 59 & 100 \\
\hline $\mathrm{S} 27$ & $08^{\circ} 48^{\prime} 00^{\prime \prime}$ & $78^{\circ} 10^{\prime} 00^{\prime \prime}$ & 8.2 & 250 & 145 & 100 & 24 & 3 & 10 & 10 & 59 & 28 & 22 & 0.35 & 2 & 34 \\
\hline S28 & $08^{\circ} 44^{\prime} 12^{\prime \prime}$ & $77^{\circ} 59^{\prime} 45^{\prime \prime}$ & 8.2 & 330 & 215 & 95 & 22 & 2 & 35 & 10 & 54 & 35 & 29 & 0.8 & 11 & 45 \\
\hline S29 & $08^{\circ} 38^{\prime} 00^{\prime \prime}$ & $77^{\circ} 55^{\prime} 00^{\prime \prime}$ & 8.4 & 660 & 391 & 170 & 34 & 13 & 69 & 21 & 151 & 82 & 48 & 0.2 & 6 & 66 \\
\hline $\mathrm{S} 30$ & $08^{\circ} 34^{\prime} 30^{\prime \prime}$ & $78^{\circ} 05^{\prime} 45^{\prime \prime}$ & 8 & 1550 & 914 & 320 & 24 & 100 & 161 & 63 & 445 & 191 & 125 & 1.45 & 6 & 178 \\
\hline S31 & $08^{\circ} 34^{\prime} 40^{\prime \prime}$ & $77^{\circ} 49^{\prime} 15^{\prime \prime}$ & 8.8 & 2530 & 1459 & 460 & 76 & 16 & 368 & 66 & 427 & 475 & 192 & 1.07 & 8 & 243 \\
\hline $\mathrm{S} 32$ & $08^{\circ} 26^{\prime} 40^{\prime \prime}$ & $78^{\circ} 00^{\prime} 32^{\prime \prime}$ & 8 & 2230 & 1302 & 240 & 48 & 18 & 391 & 29 & 702 & 255 & 192 & 1.03 & 4 & 178 \\
\hline S33 & $08^{\circ} 29^{\prime} 00^{\prime \prime}$ & $78^{\circ} 01^{\prime} 30^{\prime \prime}$ & 8 & 530 & 328 & 155 & 34 & 59 & 23 & 17 & 218 & 18 & 10 & 0.36 & 7 & 67 \\
\hline $\mathrm{S} 34$ & $09^{\circ} 05^{\prime} 00^{\prime \prime}$ & $78^{\circ} 05^{\prime} 00^{\prime \prime}$ & 8.3 & 480 & 249 & 125 & 18 & 2 & 55 & 19 & 211 & 7 & 13 & 0.86 & 1 & 43 \\
\hline $\mathrm{S} 35$ & $08^{\circ} 27^{\prime} 00^{\prime \prime}$ & $78^{\circ} 01^{\prime} 00^{\prime \prime}$ & 8.2 & 1470 & 799 & 420 & 48 & 11 & 143 & 73 & 214 & 347 & 58 & 0.6 & 3 & 164 \\
\hline S36 & $09^{\circ} 12^{\prime} 54^{\prime \prime}$ & $78^{\circ} 12^{\prime} 30^{\prime \prime}$ & 8 & 980 & 563 & 305 & 50 & 13 & 87 & 44 & 244 & 152 & 63 & 0.79 & 7 & 106 \\
\hline S37 & $08^{\circ} 25^{\prime} 00^{\prime \prime}$ & $78^{\circ} 57^{\prime} 00^{\prime \prime}$ & 7.8 & 830 & 473 & 340 & 64 & 4 & 32 & 44 & 114 & 131 & 43 & 0.16 & 19 & 86 \\
\hline S38 & $08^{\circ} 24^{\prime} 30^{\prime \prime}$ & $77^{\circ} 52^{\prime} 00^{\prime \prime}$ & 8.2 & 1090 & 616 & 300 & 50 & 7 & 115 & 42.5 & 293 & 181 & 35 & 0.74 & 9 & 142 \\
\hline
\end{tabular}

Table 7. Physico-chemical values in 2018

\begin{tabular}{c|c|c|c|c|c|c|c|c|c|c|c|c|c|c|c|c}
\hline $\begin{array}{c}\text { Well } \\
\text { No }\end{array}$ & Latitude & Longitude & $\mathbf{p H}$ & $\mathbf{E C}$ & $\mathbf{T D S}$ & $\mathbf{T H}$ & $\mathbf{C a}$ & $\mathbf{K}$ & $\mathbf{N a}$ & $\mathbf{M g}$ & $\mathbf{H C O}_{\mathbf{3}}$ & $\mathbf{C l}$ & $\mathbf{S O}_{\mathbf{4}}$ & $\mathbf{F}^{\prime}$ & $\mathbf{N O}_{\mathbf{3}}$ & $\begin{array}{c}\text { WQI } \\
\mathbf{2 0 1 8}\end{array}$ \\
\hline S1 & $09^{\circ} 13^{\prime} 47^{\prime \prime}$ & $78^{\circ} 08^{\prime} 12^{\prime \prime}$ & 8.2 & 1450 & 888 & 255 & 42 & 19 & 212 & 36 & 293 & 209 & 71 & 0.09 & 34 & 133 \\
S2 & $09^{\circ} 14^{\prime} 09^{\prime \prime}$ & $78^{\circ} 01^{\prime} 50^{\prime \prime}$ & 8.2 & 1600 & 1022 & 430 & 92 & 20 & 166 & 49 & 329 & 163 & 125 & 0.06 & 55 & 250 \\
S3 & $09^{\circ} 17^{\prime} 47^{\prime \prime}$ & $78^{\circ} 03^{\prime} 56^{\prime \prime}$ & 7.6 & 2640 & 1559 & 740 & 184 & 23 & 258 & 68 & 354 & 532 & 204 & 0.12 & 25 & 266 \\
S4 & $09^{\circ} 10^{\prime} 45^{\prime \prime}$ & $77^{\circ} 52^{\prime} 25^{\prime \prime}$ & 8.2 & 610 & 352 & 165 & 28 & 4 & 67 & 23 & 207 & 28 & 27 & 0.23 & 11 & 50 \\
S5 & $09^{\circ} 08^{\prime} 30^{\prime \prime}$ & $78^{\circ} 02^{\prime} 15^{\prime \prime}$ & 7.9 & 2680 & 1568 & 510 & 144 & 10 & 396 & 36 & 683 & 425 & 72 & 0.16 & 32 & 260 \\
S6 & $09^{\circ} 08^{\prime} 57^{\prime \prime}$ & $77^{\circ} 42^{\prime} 10^{\prime \prime}$ & 8.1 & 190 & 99 & 80 & 18 & 1 & 7 & 9 & 74 & 7 & 7 & 0.32 & 1 & 24 \\
S7 & $09^{\circ} 09^{\prime} 00^{\prime \prime}$ & $77^{\circ} 59^{\prime} 54^{\prime \prime}$ & 8.8 & 2040 & 1235 & 200 & 40 & 16 & 368 & 24 & 500 & 220 & 125 & 0.08 & 39 & 155 \\
S8 & $09^{\circ} 07^{\prime} 45^{\prime \prime}$ & $78^{\circ} 10^{\prime} 07^{\prime \prime}$ & 8.2 & 3180 & 1886 & 670 & 72 & 47 & 414 & 119 & 268 & 737 & 250 & 0.06 & 25 & 335 \\
S9 & $09^{\circ} 08^{\prime} 40^{\prime \prime}$ & $77^{\circ} 47^{\prime} 15^{\prime \prime}$ & 8.1 & 310 & 188 & 105 & 26 & 5 & 23 & 10 & 69 & 35 & 10 & 0.4 & 8 & 40 \\
S10 & $0^{\circ} 04^{\prime} 30^{\prime \prime}$ & $77^{\circ} 48^{\prime} 30^{\prime \prime}$ & 8.2 & 920 & 555 & 210 & 46 & 7 & 113 & 23 & 299 & 67 & 42 & 0.17 & 24 & 76 \\
S11 & $09^{\circ} 06^{\prime} 00^{\prime \prime}$ & $78^{\circ} 00^{\prime} 40^{\prime \prime}$ & 7.4 & 1890 & 1138 & 620 & 148 & 14 & 166 & 61 & 256 & 383 & 61 & 0.21 & 40 & 201 \\
S12 & $0^{\circ} 33^{\prime} 00^{\prime \prime}$ & $78^{\circ} 01^{\prime} 00^{\prime \prime}$ & 7.9 & 1050 & 686 & 370 & 94 & 11 & 74 & 33 & 153 & 96 & 216 & 0.21 & 19 & 99 \\
S13 & $09^{\circ} 04^{\prime} 00^{\prime \prime}$ & $78^{\circ} 10^{\prime} 35^{\prime \prime}$ & 8.1 & 9310 & 5581 & 1200 & 100 & 7 & 1610 & 231 & 732 & 1914 & 1344 & 0.85 & 2 & 837
\end{tabular}




\begin{tabular}{|c|c|c|c|c|c|c|c|c|c|c|c|c|c|c|c|c|}
\hline S14 & $09^{\circ} 13^{\prime} 47^{\prime \prime}$ & $78^{\circ} 08^{\prime} 12^{\prime \prime}$ & 8 & 5680 & 3268 & 1420 & 272 & 8 & 644 & 180 & 708 & 993 & 768 & 0.25 & 11 & 502 \\
\hline S15 & $09^{\circ} 00^{\prime} 10^{\prime \prime}$ & $78^{\circ} 11^{\prime} 50^{\prime \prime}$ & 8.1 & 3590 & 2125 & 660 & 40 & 11 & 552 & 136 & 403 & 695 & 456 & 0.92 & 8 & 338 \\
\hline S16 & $09^{\circ} 03^{\prime} 35^{\prime \prime}$ & $77^{\circ} 44^{\prime} 50^{\prime \prime}$ & 8.1 & 870 & 514 & 90 & 12 & 16 & 154 & 15 & 360 & 39 & 83 & 1.43 & 3 & 78 \\
\hline S17 & $08^{\circ} 59^{\prime} 40^{\prime \prime}$ & $77^{\circ} 51^{\prime} 38^{\prime \prime}$ & 8 & 120 & 73 & 40 & 8 & 7 & 7 & 49 & 35 & 14 & 6 & 0.43 & 1 & 32 \\
\hline S18 & $09^{\circ} 00^{\prime} 00^{\prime \prime}$ & $77^{\circ} 58^{\prime} 00^{\prime \prime}$ & 8.2 & 3150 & 1885 & 580 & 112 & 4 & 460 & 73 & 458 & 596 & 206 & 0.06 & 46 & 287 \\
\hline S19 & $09^{\circ} 03^{\prime} 00^{\prime \prime}$ & $77^{\circ} 54^{\prime} 00^{\prime \prime}$ & 8 & 250 & 145 & 100 & 24 & 7 & 10 & 10 & 99 & 7 & 10 & 0.07 & 4 & 28 \\
\hline S20 & $09^{\circ} 01^{\prime} 10^{\prime \prime}$ & $78^{\circ} 02^{\prime} 45^{\prime \prime}$ & 8.7 & 2970 & 1826 & 320 & 44 & 20 & 552 & 51 & 598 & 319 & 451 & 1.4 & 16 & 235 \\
\hline S21 & $08^{\circ} 56^{\prime} 40^{\prime \prime}$ & $77^{\circ} 52^{\prime} 00^{\prime \prime}$ & 8.2 & 1910 & 1136 & 340 & 32 & 3 & 294 & 63 & 500 & 248 & 165 & 0.51 & 18 & 160 \\
\hline S22 & $08^{\circ} 54^{\prime} 45^{\prime \prime}$ & $78^{\circ} 01^{\prime} 25^{\prime \prime}$ & 7.5 & 3380 & 1994 & 740 & 144 & 7 & 460 & 92 & 647 & 560 & 202 & 0.13 & 46 & 297 \\
\hline S23 & $08^{\circ} 52^{\prime} 30^{\prime \prime}$ & $78^{\circ} 02^{\prime} 15^{\prime \prime}$ & 8.1 & 1780 & 1068 & 490 & 68 & 19 & 173 & 78 & 281 & 305 & 160 & 0.12 & 28 & 175 \\
\hline S24 & $08^{\circ} 51^{\prime} 38^{\prime \prime}$ & $77^{\circ} 54^{\prime} 00^{\prime \prime}$ & 8.2 & 1380 & 786 & 250 & 54 & 5 & 207 & 28 & 488 & 152 & 71 & 0.11 & 6 & 108 \\
\hline S25 & $08^{\circ} 47^{\prime} 25^{\prime \prime}$ & $77^{\circ} 52^{\prime} 15^{\prime \prime}$ & 8.2 & 340 & 194 & 125 & 18 & 9 & 20 & 19 & 89 & 39 & 24 & 0.83 & 2 & 48 \\
\hline S26 & $08^{\circ} 48^{\prime} 35^{\prime \prime}$ & $78^{\circ} 01^{\prime} 25^{\prime \prime}$ & 8.2 & 990 & 599 & 230 & 24 & 63 & 92 & 41 & 256 & 121 & 84 & 0.46 & 10 & 114 \\
\hline S27 & $08^{\circ} 48^{\prime} 00^{\prime \prime}$ & $78^{\circ} 10^{\prime} 00^{\prime \prime}$ & 8.1 & 8410 & 5350 & 2120 & 36 & 7 & 1104 & 493 & 488 & 1773 & 1440 & 1.31 & 28 & 840 \\
\hline S28 & $08^{\circ} 44^{\prime} 12^{\prime \prime}$ & $77^{\circ} 59^{\prime} 45^{\prime \prime}$ & 8 & 970 & 560 & 210 & 18 & 6 & 124 & 40 & 244 & 121 & 83 & 0.4 & 10 & 88 \\
\hline S29 & $08^{\circ} 38^{\prime} 00^{\prime \prime}$ & $77^{\circ} 55^{\prime} 00^{\prime \prime}$ & 8.1 & 340 & 189 & 125 & 30 & 3 & 20 & 12 & 89 & 35 & 18 & 0.39 & 4 & 40 \\
\hline S30 & $08^{\circ} 34^{\prime} 30^{\prime \prime}$ & $78^{\circ} 05^{\prime} 45^{\prime \prime}$ & 8.2 & 1360 & 796 & 315 & 42 & 59 & 143 & 51 & 439 & 145 & 80 & 1.33 & 13 & 145 \\
\hline S31 & $08^{\circ} 34^{\prime} 40^{\prime \prime}$ & $77^{\circ} 49^{\prime} 15^{\prime \prime}$ & 8.2 & 840 & 499 & 100 & 12 & 4 & 147 & 17 & 217 & 60 & 62 & 0.66 & 14 & 68 \\
\hline S32 & $08^{\circ} 26^{\prime} 40^{\prime \prime}$ & $78^{\circ} 00^{\prime} 32^{\prime \prime}$ & 8.2 & 2140 & 1227 & 500 & 112 & 9 & 276 & 53 & 525 & 376 & 112 & 0.35 & 6 & 199 \\
\hline S33 & $08^{\circ} 29^{\prime} 00^{\prime \prime}$ & $78^{\circ} 01^{\prime} 30^{\prime \prime}$ & 7.8 & 1390 & 835 & 445 & 120 & 8 & 117 & 35 & 177 & 273 & 75 & 0.2 & 27 & 147 \\
\hline S34 & $09^{\circ} 05^{\prime} 00^{\prime \prime}$ & $78^{\circ} 05^{\prime} 00^{\prime \prime}$ & 8.2 & 490 & 286 & 210 & 54 & 4 & 20 & 18 & 108 & 60 & 50 & 0.32 & 3 & 55 \\
\hline S35 & $08^{\circ} 27^{\prime} 00^{\prime \prime}$ & $78^{\circ} 01^{\prime} 00^{\prime \prime}$ & 8 & 2150 & 1288 & 300 & 36 & 63 & 336 & 51 & 580 & 305 & 81 & 1.28 & 28 & 211 \\
\hline S36 & $09^{\circ} 12^{\prime} 54^{\prime \prime}$ & $78^{\circ} 12^{\prime} 30^{\prime \prime}$ & 8 & 470 & 274 & 110 & 16 & 6 & 58 & 17 & 129 & 53 & 30 & 0.09 & 3 & 46 \\
\hline S37 & $08^{\circ} 25^{\prime} 00^{\prime \prime}$ & $78^{\circ} 57^{\prime} 00^{\prime \prime}$ & 8.2 & 780 & 441 & 220 & 42 & 16 & 74 & 28 & 197 & 106 & 26 & 1.03 & 6 & 87 \\
\hline S38 & $08^{\circ} 24^{\prime} 30^{\prime \prime}$ & $77^{\circ} 52^{\prime} 00^{\prime \prime}$ & 8.2 & 1350 & 816 & 305 & 58 & 11 & 173 & 39 & 171 & 266 & 78 & 0.44 & 24 & 142 \\
\hline
\end{tabular}

The water quality characteristics of the groundwater samples during the year 2014 and 2018 is statistically analysed and the results such as maximum, minimum, mean and standard deviation values are given in Table 8.

Table 8. Statistical measures such as minimum, maximum, mean and standard deviation in 2014 and 2018

\begin{tabular}{c|c|c|c|c|c|c|c|c}
\hline \multirow{2}{*}{$\begin{array}{c}\text { Water quality } \\
\text { parameters }\end{array}$} & \multicolumn{9}{|c|}{ Jan 2014 } & \multicolumn{4}{c}{ Jan 2018 } \\
\cline { 2 - 9 } & Min & Max & Mean & SD & Min & Max & Mean & SD \\
\hline $\mathrm{pH}$ & 7.4 & 8.8 & 8.09 & 0.25 & 7.5 & 8.8 & 8.12 & 0.38 \\
$\mathrm{EC}$ & 120 & 9310 & 1972.89 & 2028.29 & 250 & 4180 & 1662.11 & 1045.56 \\
$\mathrm{HCO} 3$ & 35 & 732 & 328.93 & 199.79 & 54 & 702 & 311.63 & 175.17 \\
$\mathrm{TDS}$ & 73 & 5581 & 1182.39 & 1236.79 & 145 & 2503 & 996.74 & 637.78 \\
$\mathrm{~F}$ & 0 & 1 & 0.46 & 0.43 & 0 & 2 & 0.64 & 0.42 \\
$\mathrm{Cl}$ & 7 & 1914 & 328.34 & 430.94 & 7 & 865 & 253.03 & 224.93 \\
$\mathrm{NO}$ & 1 & 55 & 17.89 & 14.64 & 1 & 73 & 19.42 & 20.54 \\
$\mathrm{SO}$ & 6 & 1440 & 194.08 & 322.85 & 10 & 624 & 145.63 & 133.62 \\
$\mathrm{Na}$ & 7 & 1610 & 265.55 & 317.25 & 10 & 552 & 196.18 & 154.98 \\
$\mathrm{Ca}$ & 8 & 272 & 64.79 & 56.65 & 8 & 360 & 74.53 & 75.39 \\
$\mathrm{Mg}$ & 5 & 493 & 62.32 & 86.10 & 10 & 175 & 52.34 & 31.98 \\
$\mathrm{~K}$ & 1 & 63 & 14.71 & 16.17 & 1 & 121 & 24.84 & 33.06 \\
$\mathrm{TH}$ & 40 & 2120 & 418.42 & 412.23 & 95 & 1320 & 401.71 & 277.38 \\
\hline
\end{tabular}

The values of water quality characteristics like $\mathrm{pH}, \mathrm{EC}, \mathrm{HCO}_{3}^{-}, \mathrm{TDS}, \mathrm{F}^{-}, \mathrm{Cl}^{-}, \mathrm{NO}_{3}^{-}$, $\mathrm{SO}_{4}{ }^{2-}, \mathrm{Na}^{+}, \mathrm{Ca}^{2+}, \mathrm{Mg}^{2+}, \mathrm{K}^{+}$and $\mathrm{TH}$ in 2014 and 2018 are compared with drinking water quality norms recommended by WHO (2017) and the samples beyond allowable limit during the years 2014 and 2018 are given in Table 9. 
Table 9. Comparison of groundwater quality with WHO standards

\begin{tabular}{c|c|c|c|c|c}
\hline \multirow{2}{*}{$\begin{array}{c}\text { Water quality } \\
\text { parameter }\end{array}$} & Units & \multicolumn{2}{|c|}{ WHO (2017) } & $\begin{array}{c}\text { Samples exceeding } \\
\text { allowable limit in } \\
\mathbf{2 0 1 4}\end{array}$ & $\begin{array}{c}\text { Samples exceeding } \\
\text { allowable limit in } \\
\mathbf{2 0 1 8}\end{array}$ \\
\cline { 3 - 5 } & $\begin{array}{c}\text { Most desirable } \\
\text { limit }\end{array}$ & $\begin{array}{c}\text { Maximum } \\
\text { allowable limit }\end{array}$ & 2 & 6 \\
$\mathrm{pH}$ & - & 6.5 & 8.5 & 17 & 19 \\
$\mathrm{EC}$ & $\mu \mathrm{s} / \mathrm{cm}$ & 780 & 3125 & 16 & 20 \\
$\mathrm{HCO}_{3}$ & $\mathrm{mg} / \mathrm{L}$ & - & 300 & 16 & 17 \\
$\mathrm{TDS}$ & $\mathrm{mg} / \mathrm{L}$ & 500 & 1500 & 1 & 0 \\
$\mathrm{~F}$ & $\mathrm{mg} / \mathrm{L}$ & - & 1.5 & 11 & 13 \\
$\mathrm{Cl}$ & $\mathrm{mg} / \mathrm{L}$ & 200 & 600 & 3 & 5 \\
$\mathrm{NO}$ & $\mathrm{mg} / \mathrm{L}$ & 45 & - & 2 & 16 \\
$\mathrm{SO}$ & $\mathrm{mg} / \mathrm{L}$ & 200 & 400 & 15 & 1 \\
$\mathrm{Na}$ & $\mathrm{mg} / \mathrm{L}$ & - & 200 & 3 & 3 \\
$\mathrm{Ca}$ & $\mathrm{mg} / \mathrm{L}$ & 75 & 200 & 1 & 16 \\
$\mathrm{Mg}$ & $\mathrm{mg} / \mathrm{L}$ & 30 & 150 & 20 & 8 \\
$\mathrm{~K}$ & $\mathrm{mg} / \mathrm{L}$ & - & 10 & 5 & 5 \\
$\mathrm{TH}$ & $\mathrm{mg} / \mathrm{L}$ & 300 & 600 & & 2 \\
\hline
\end{tabular}

\section{$p H$}

A $\mathrm{pH}$ value 7 indicates neutral. If the value is lower than 7 it indicates acidity and higher than 7 indicates alkalinity. The values of $\mathrm{pH}$ ranges from 7.5 to 8.8 and 7.4 to 8.8 during 2014 and 2018 which specifies that the groundwater is alkaline in both 2014 and 2018 and some of the samples exceed the maximum permissible limits of WHO standards. The slight alkalinity is due to the existence of bicarbonate ions, which are formed by the free combination of $\mathrm{CO}_{2}$ in water with the carbonates from the aquatic life, which affects the $\mathrm{pH}$ of the water (Azeez et al., 2000). The spatial distribution of $\mathrm{pH}$ is shown in Figure 4 indicates all the samples are within the permissible limit of 6.58.5., except two samples in 2014 and six samples in 2018.
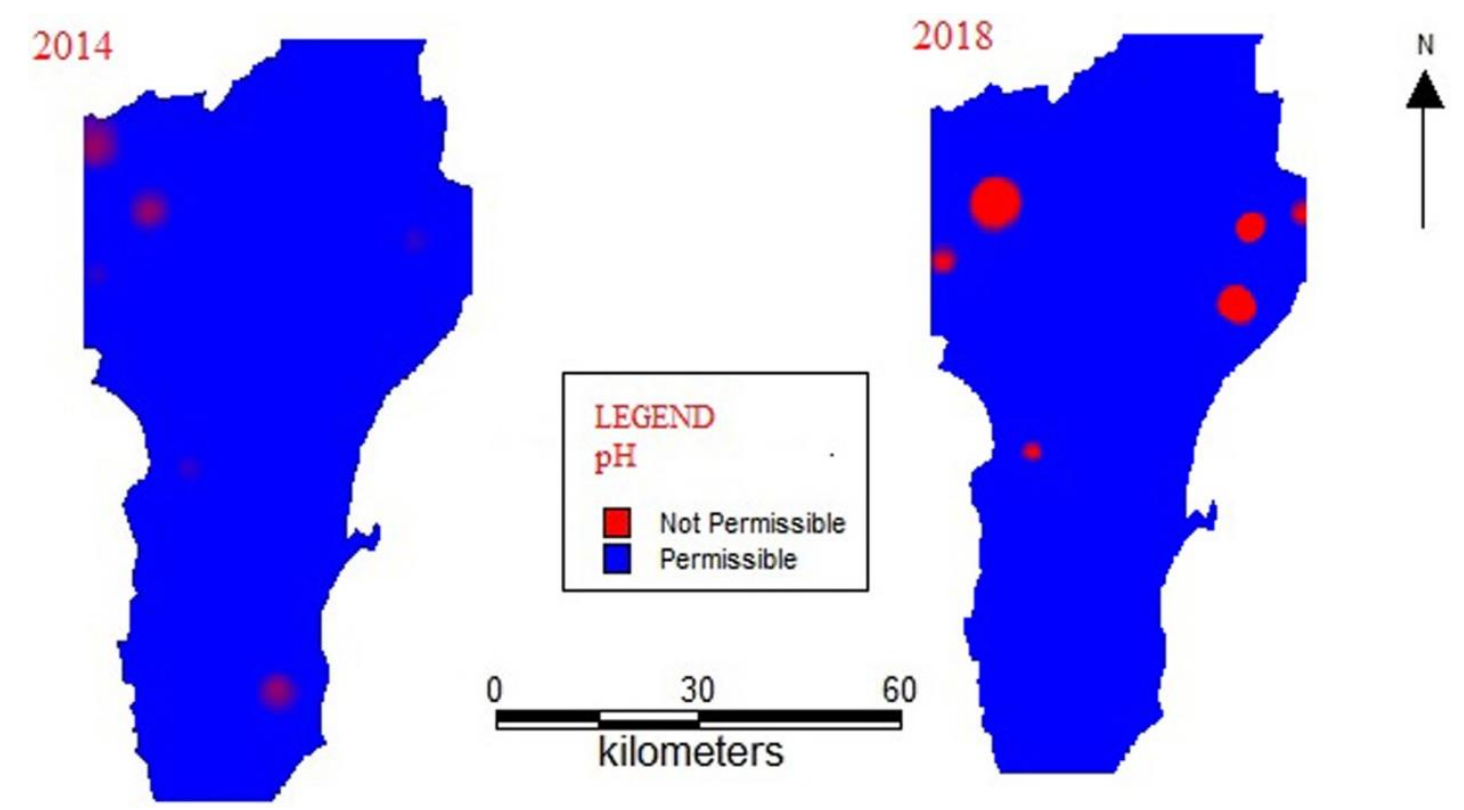

Figure 4. Spatial distribution of $p H$ 


\section{Electrical conductivity}

Electrical conductivity is the most significant parameter to delineate salinity hazard and suitability of water for irrigation purpose. The EC vary from 250 to $4180 \mu \mathrm{s} / \mathrm{cm}$ and from 120 to $9310 \mu \mathrm{s} / \mathrm{cm}$ during the years 2014 and 2018, respectively. The spatial distribution of EC is shown in Figure 5. Higher value of EC were noted during 2018 when compared to that of 2014. The classification of water salinity based upon Electrical conductivity shows that $45 \%$ in 2014 and 50\% in 2018 falls within the permissible limit. To determine the salinity of groundwater, it is important to categorize the groundwater based on their EC values (Handa, 1969), which are represented in Table 10.

Table 10. Classification of waters based on EC (Handa, 1969)

\begin{tabular}{|c|c|c|c|c|c|}
\hline \multirow{2}{*}{$\begin{array}{c}\mathrm{EC} \\
(\mu \mathrm{s} / \mathrm{cm})\end{array}$} & \multirow[b]{2}{*}{ Water salinity } & \multicolumn{2}{|c|}{2014} & \multicolumn{2}{|c|}{2018} \\
\hline & & $\begin{array}{l}\text { Number of } \\
\text { samples }\end{array}$ & $\begin{array}{l}\text { Percentage } \\
\text { of samples }\end{array}$ & $\begin{array}{c}\text { Number of } \\
\text { samples }\end{array}$ & $\begin{array}{l}\text { Percentage } \\
\text { of samples }\end{array}$ \\
\hline $0-250$ & Low & 1 & 3 & 3 & 8 \\
\hline $251-750$ & Medium & 9 & 24 & 6 & 16 \\
\hline $751-2250$ & High & 17 & 45 & 19 & 50 \\
\hline $2251-6000$ & Very high & 11 & 20 & 8 & 21 \\
\hline $6001-10000$ & Extremely high & - & - & 2 & 5 \\
\hline $10001-20000$ & Brines weakly concentrated & - & - & - & - \\
\hline $20001-50000$ & Brines moderately concentrated & - & - & - & - \\
\hline $50001-100000$ & Brines highly concentrated & - & - & - & - \\
\hline$>100000$ & $\begin{array}{l}\text { Brines extremely highly } \\
\text { concentrated }\end{array}$ & - & - & - & - \\
\hline Total & & 38 & 100 & 38 & 100 \\
\hline
\end{tabular}
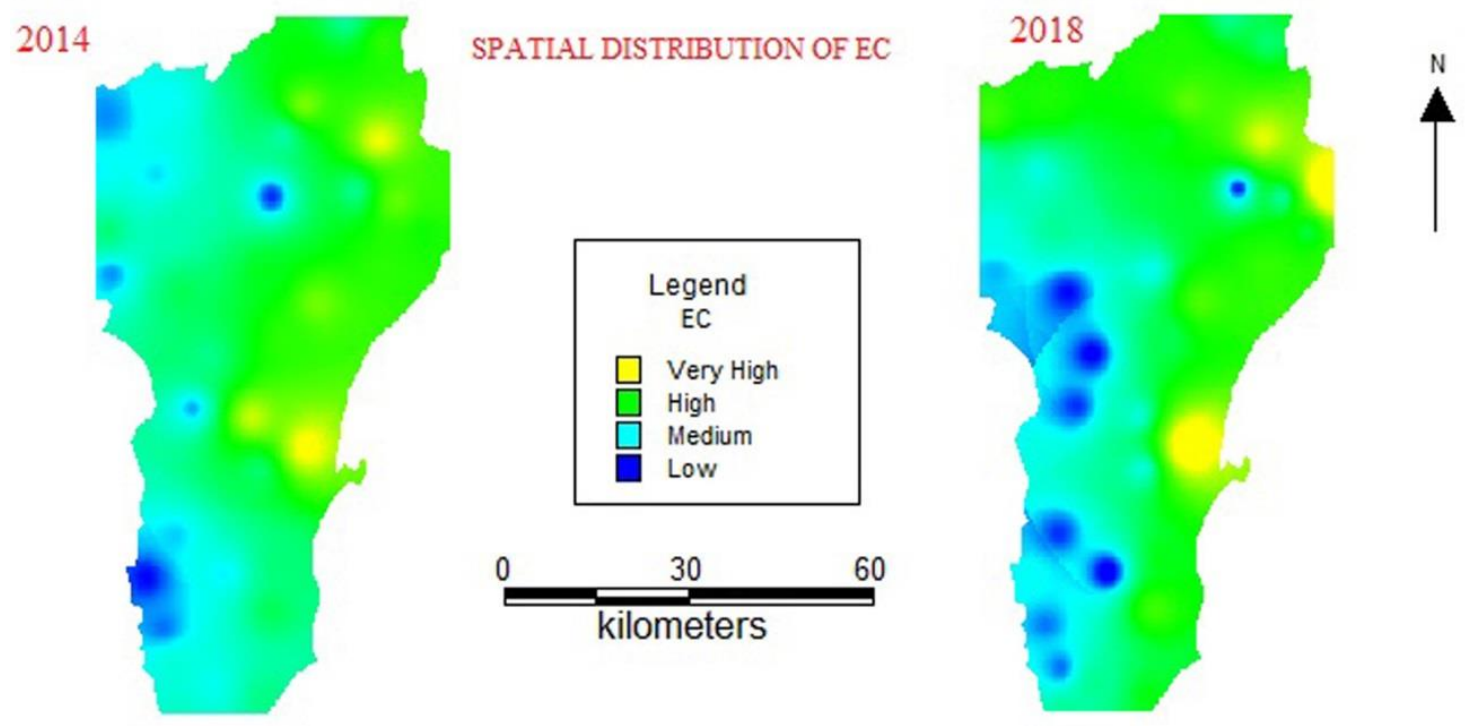

Figure 5. Spatial distribution of EC 


\section{Total dissolved solids}

TDS of the groundwater are found to vary from 145 to $2503 \mathrm{mg} / \mathrm{L}$ during 2014 and 73 to $5581 \mathrm{mg} / \mathrm{L}$ during 2018. The Spatial Distribution of TDS is shown in Figure 6. Some groundwater samples having high value of TDS are may be due to presence of excess salts in the soil and also by manmade activities. Sewage waste from household may penetrate into the groundwater which also leads to raise in TDS values. To ascertain the groundwater, it is essential to categorize the groundwater based on their TDS values, which are presented in Tables 11 and 12, respectively.

Table 11. Classification of groundwater based on TDS (Davis, 1966)

\begin{tabular}{c|c|c|c|c|c}
\hline \multirow{2}{*}{$\begin{array}{c}\text { Total dissolved } \\
\text { solids (mg/L) }\end{array}$} & Classification & \multicolumn{2}{|c|}{$\mathbf{2 0 1 4}$} & \multicolumn{2}{|c|}{$\mathbf{2 0 1 8}$} \\
\cline { 3 - 6 } & & $\begin{array}{c}\text { Number of } \\
\text { samples }\end{array}$ & $\begin{array}{c}\text { Percentage } \\
\text { of samples }\end{array}$ & $\begin{array}{c}\text { Number of } \\
\text { samples }\end{array}$ & $\begin{array}{c}\text { Percentage } \\
\text { of samples }\end{array}$ \\
\hline$<500$ & Desirable for drinking & 12 & 32 & 11 & 32 \\
$500-1000$ & Permissible for drinking & 10 & 26 & 10 & 26 \\
$1000-3000$ & Useful for irrigation & 16 & 42 & 14 & 37 \\
$>3000$ & Unfit for drinking and irrigation & 0 & - & 3 & 8 \\
Total & & 38 & 100 & 38 & 100 \\
\hline
\end{tabular}

Table 12. Classification of groundwater based on TDS (Freeze and Cherry, 1979)

\begin{tabular}{c|c|c|c|c|c}
\hline \multirow{2}{*}{$\begin{array}{c}\text { Total dissolved } \\
\text { solids (mg/L) }\end{array}$} & Classification & \multicolumn{2}{|c|}{$\mathbf{2 0 1 4}$} & \multicolumn{2}{c}{$\mathbf{2 0 1 8}$} \\
\cline { 3 - 6 } & Number of & $\begin{array}{c}\text { Percentage } \\
\text { of samples }\end{array}$ & $\begin{array}{c}\text { Number of } \\
\text { samples }\end{array}$ & $\begin{array}{c}\text { Percentage } \\
\text { of samples }\end{array}$ \\
\hline$<1000$ & Freshwater & 16 & 42 & 15 & 39 \\
$1000-10000$ & Brackish water & 22 & 58 & 23 & 61 \\
$10000-100000$ & Saline water & - & - & - & - \\
$>100000$ & Brine water & - & - & - & - \\
Total & & 38 & 100 & 38 & 100 \\
\hline
\end{tabular}
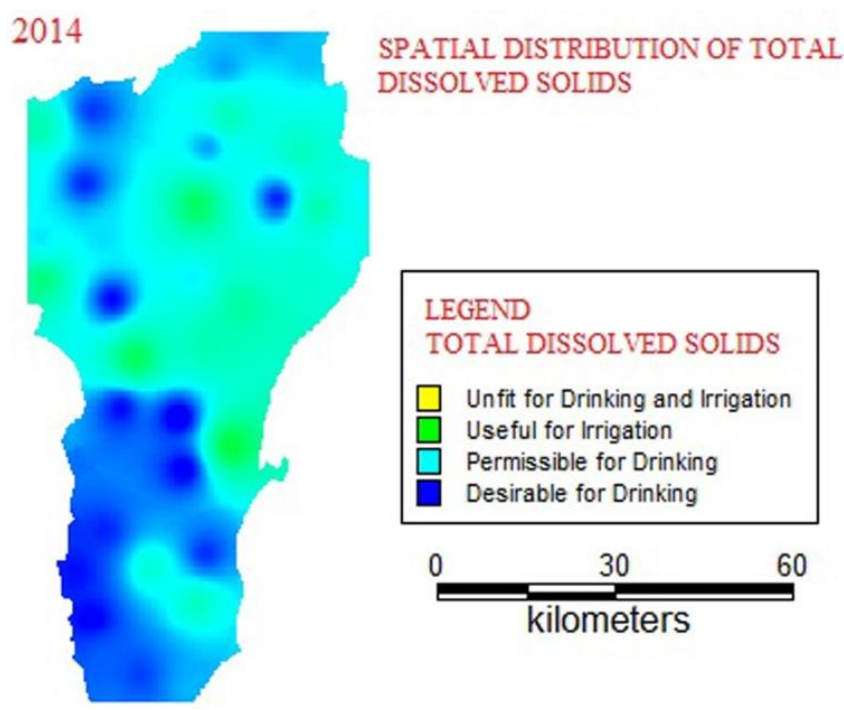
DISSOLVED SOLIDS
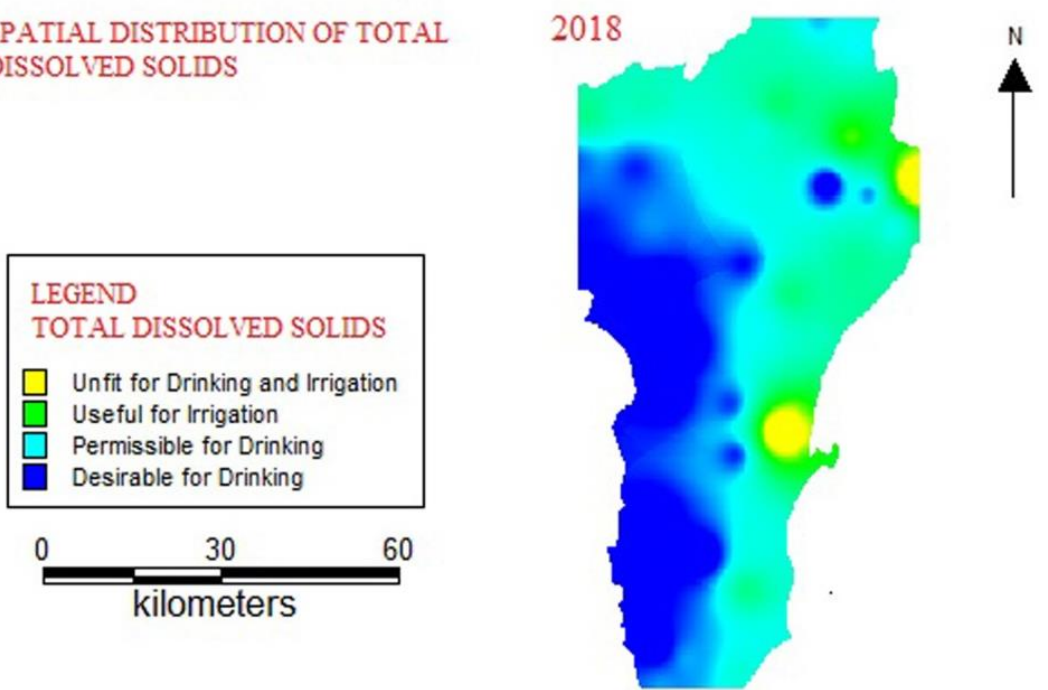

Figure 6. Spatial distribution of TDS 


\section{Total hardness}

Total hardness is found to vary from 95 to $1320 \mathrm{mg} / \mathrm{L}$ with a mean value of $401.71 \mathrm{mg} / \mathrm{L}$ during 2014 and 40 to $2120 \mathrm{mg} / \mathrm{L}$ during 2018. According to WHO, the allowable limit of TH for drinking is $600 \mathrm{mg} / \mathrm{L}$ and the desirable limit is $300 \mathrm{mg} / \mathrm{L}$. The Spatial Distribution of TH is as shown in Figure 7. The Total Hardness of the groundwater sample in the study area fell in the hard and very hard water category. Total Hardness means both temporary and permanent hardness. Hardness is a significant parameter in determining the need for industries and domestic purposes. Hard water does not produce much bad effect but it requires more detergent for cleaning and in some cases very hard water might be the reason for heart disease (Schoeller, 1965). The TH in $\mathrm{mg} / \mathrm{L}$ is determined by Equation 5 (Todd, 1959):

$$
\operatorname{TH}(\mathrm{mg} / \mathrm{L})=2: 497 \mathrm{Ca} 2^{+}+4: 115 \mathrm{Mg} 2^{+}
$$

Based on hardness groundwater is classified (Sawyer and McCarthy, 1967) and is presented in Table 13. In the study area, 22 samples were found in very hard class in 2014, whereas in 2018, 19 samples were found in very hard class. This reveals that Tuticorin experiences very hard water during both the years. High levels of hardness may affect water supply system, excessive detergent consumption and also health problem (Bhawan and Nagar, 2008).

Table 13. Classification based on hardness (Sawyer and McCarthy, 1967)

\begin{tabular}{c|c|c|c|c|c}
\hline \multirow{2}{*}{$\begin{array}{c}\text { Total hardness as } \\
\text { CaCo3 }(\mathbf{m g} / \mathbf{L})\end{array}$} & Classification & \begin{tabular}{c} 
2014 \\
Number of \\
\cline { 3 - 6 }
\end{tabular} & $\begin{array}{c}\text { Percentage } \\
\text { of samples }\end{array}$ & $\begin{array}{c}\text { Number of } \\
\text { samples }\end{array}$ & $\begin{array}{c}\text { Percentage } \\
\text { of samples }\end{array}$ \\
\hline$<75$ & Soft & 0 & 0 & 1 & 3 \\
$75-150$ & Moderately high & 3 & 8 & 8 & 21 \\
$150-300$ & Hard & 12 & 32 & 9 & 24 \\
$>300$ & Very Hard & 22 & 58 & 19 & 50 \\
Total & & 38 & 100 & 38 & 100 \\
\hline
\end{tabular}
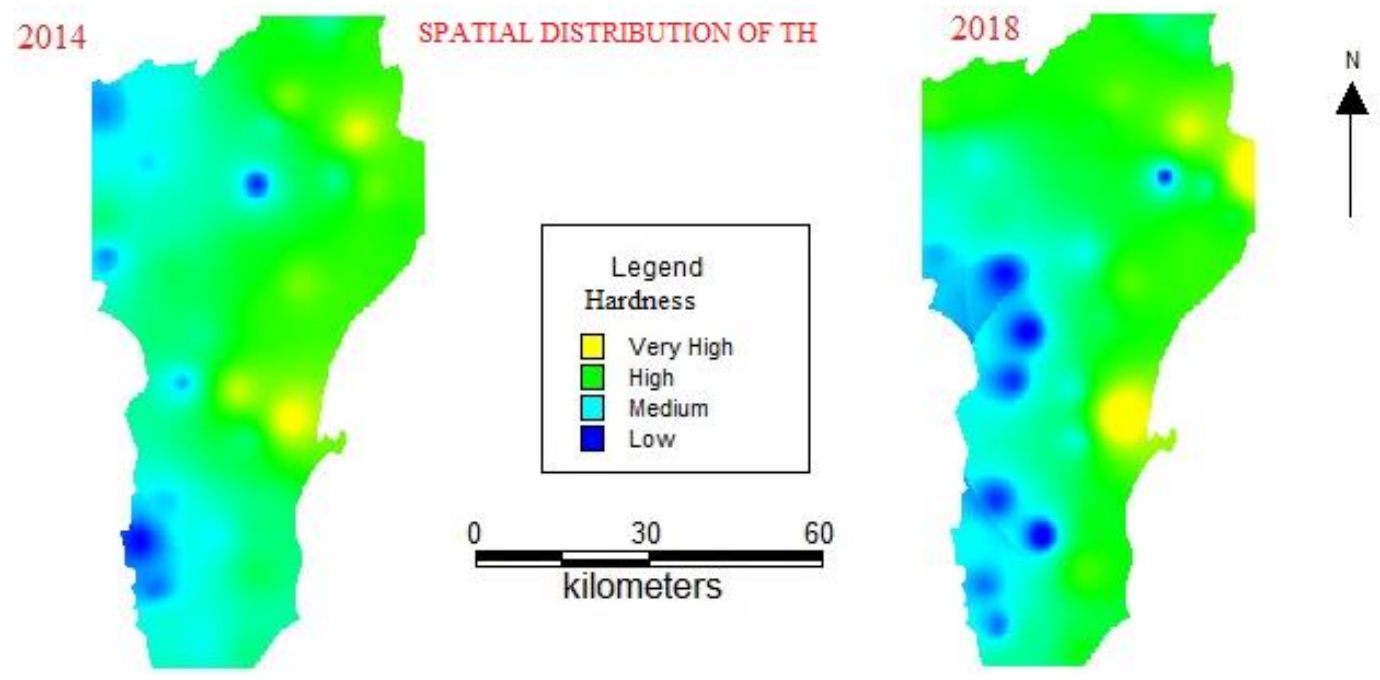

Figure 7. Spatial distribution of TH 


\section{Calcium}

Calcium concentration is found to vary between $8-360 \mathrm{mg} / \mathrm{L}$ with a mean value of $74.53 \mathrm{mg} / \mathrm{L}$ for 2014 water samples and $8-272 \mathrm{mg} / \mathrm{L}$ with a mean value of $64.79 \mathrm{mg} / \mathrm{L}$ for 2018 samples. The allowable limit of calcium in groundwater is $200 \mathrm{mg} / \mathrm{L}$ as per WHO (2017). The Spatial Distribution of Calcium is shown in Figure 8. In 2014, 92\% and in $2018,97 \%$ of samples are up to the standard and only $8 \%$ samples exceed the permissible limit in 2014 and 3\% samples exceed the permissible limit.
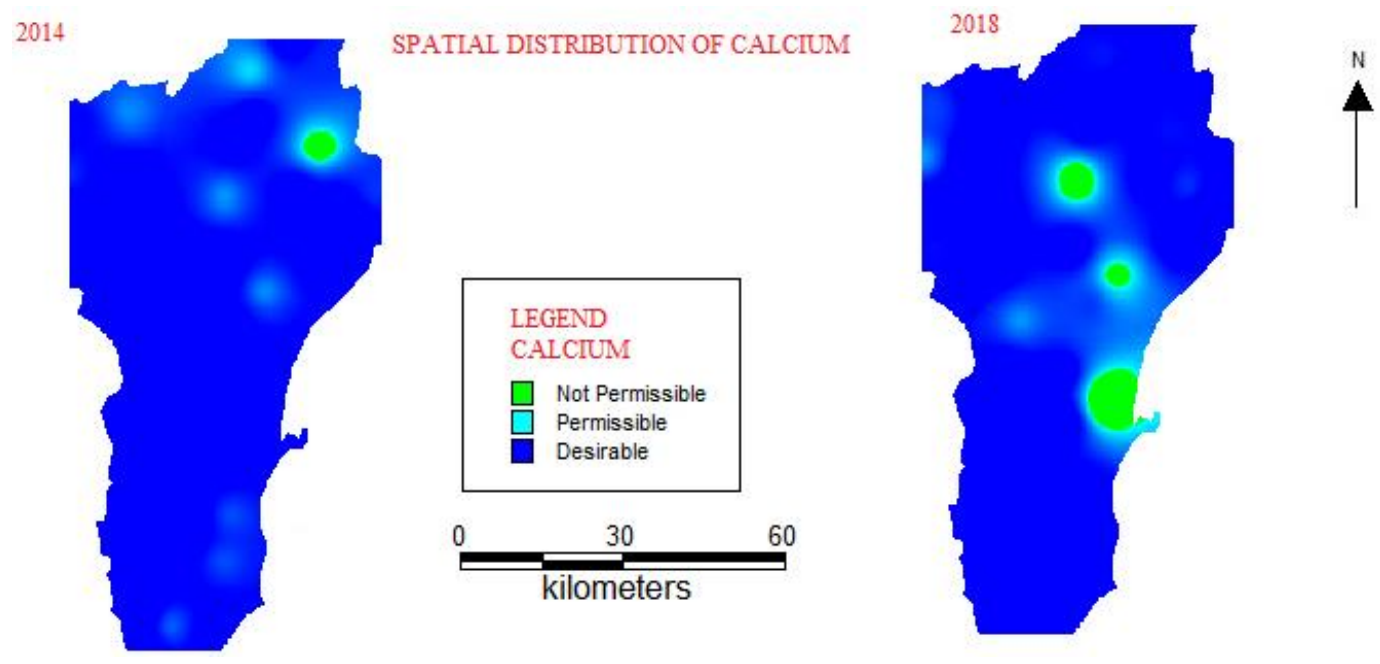

Figure 8. Spatial distribution of calcium

\section{Magnesium}

Magnesium vary from 10 to $175 \mathrm{mg} / \mathrm{L}$ with a mean value of $52.34 \mathrm{mg} / \mathrm{L}$ during the year 2014 and 5 to $493 \mathrm{mg} / \mathrm{L}$ with a mean value of $62.32 \mathrm{mg} / \mathrm{L}$ during the year 2018 . The maximum allowable limit of magnesium in groundwater is $150 \mathrm{mg} / \mathrm{L}$ as per WHO (2017). The Spatial Distribution of Magnesium is shown in Figure 9. In 2014, only 3\% of sample exceeds the permissible limit while in 2018 the $3 \%$ increased to $8 \%$ which revealed changes in water quality.
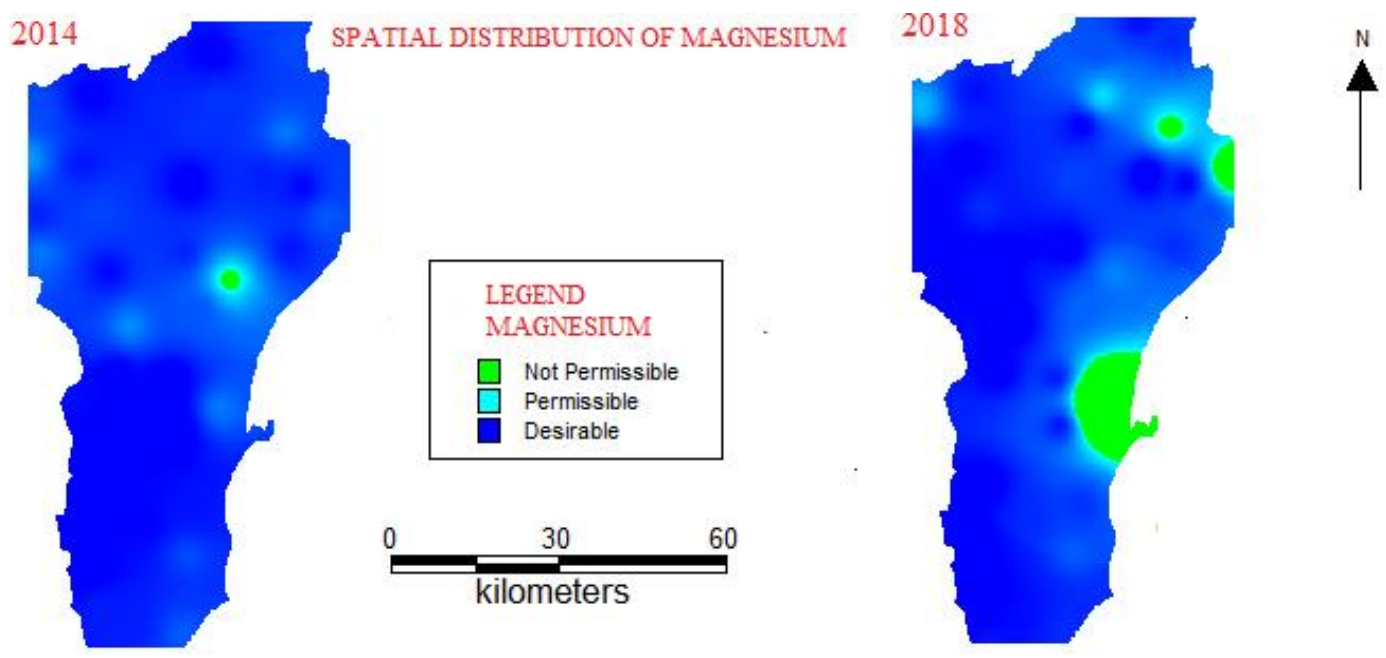

Figure 9. Spatial distribution of magnesium 


\section{Sodium}

Sodium is found to vary from 10 to $552 \mathrm{mg} / \mathrm{L}$ with a mean value of $196.18 \mathrm{mg} / \mathrm{L}$ during the year 2014 and 7 to $1610 \mathrm{mg} / \mathrm{L}$ with a mean value of $265.55 \mathrm{mg} / \mathrm{L}$ during the year 2018. Sodium causes an increase in the hardness of soil as well as decrease its permeability (Tijani, 1994). The spatial distribution of sodium is shown in Figure 10. In 2014 and 2018 the high concentration of sodium is observed which may be due to saltwater intrusion.
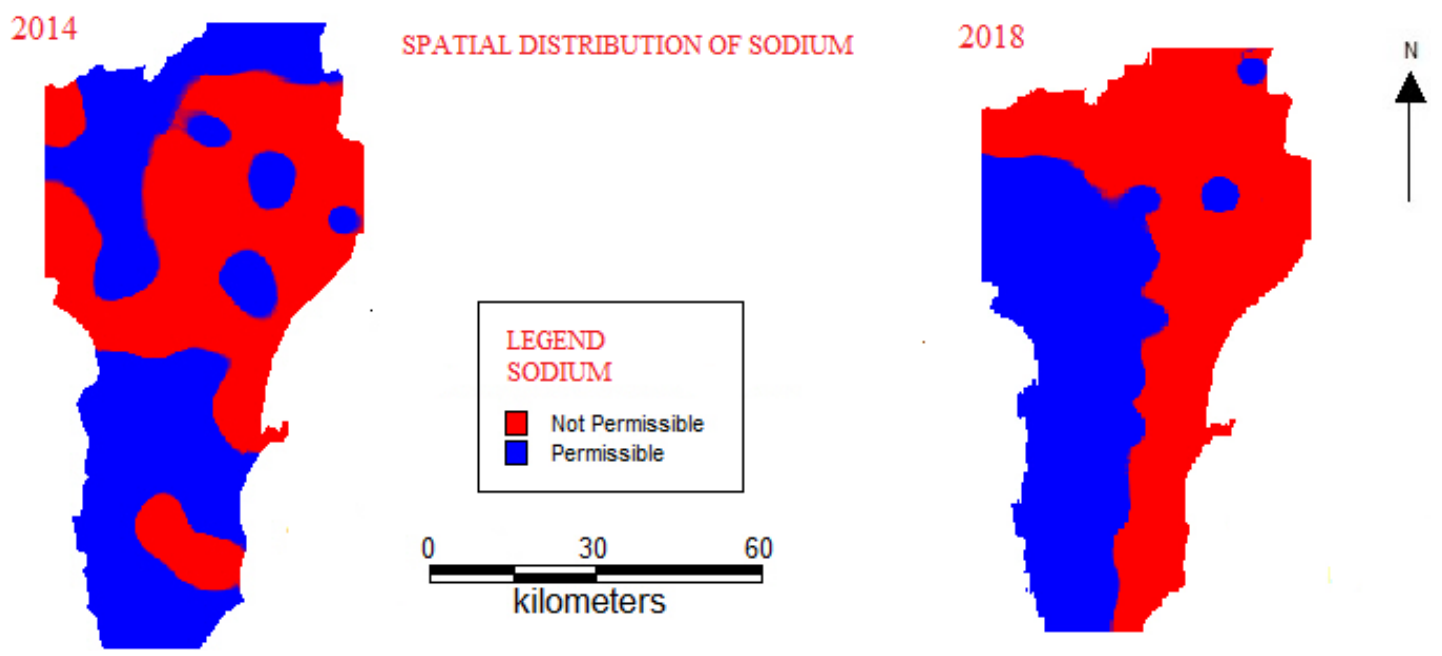

Figure 10. Spatial distribution of sodium

\section{Potassium}

Potassium ranges vary from 5 to $121 \mathrm{mg} / \mathrm{L}$ with a mean value of $24.84 \mathrm{mg} / \mathrm{L}$ during the year 2014 and 1 to $63 \mathrm{mg} / \mathrm{L}$ with a mean value of $14.71 \mathrm{mg} / \mathrm{L}$ during the year 2018 . As per WHO (2017) standards, the allowable limit for potassium is $10 \mathrm{mg} / \mathrm{L}$. The Spatial Distribution of Potassium is shown in Figure 11. Due to Salt pans, chemical fertilizer industries potassium concentration may be increased in the groundwater.

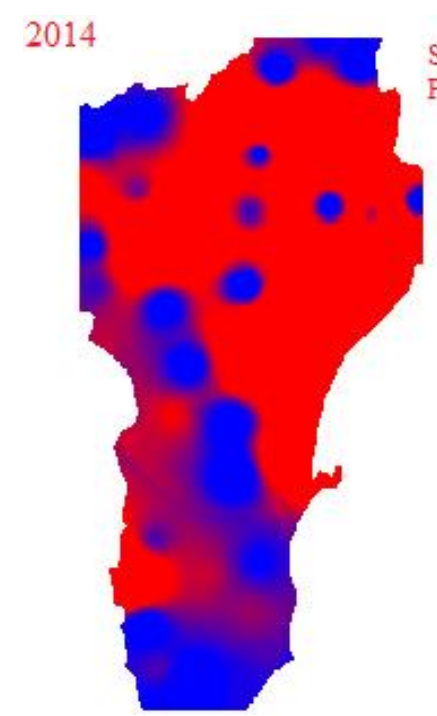

SPATIAL DISTRIBUTION OF POTASSIUM
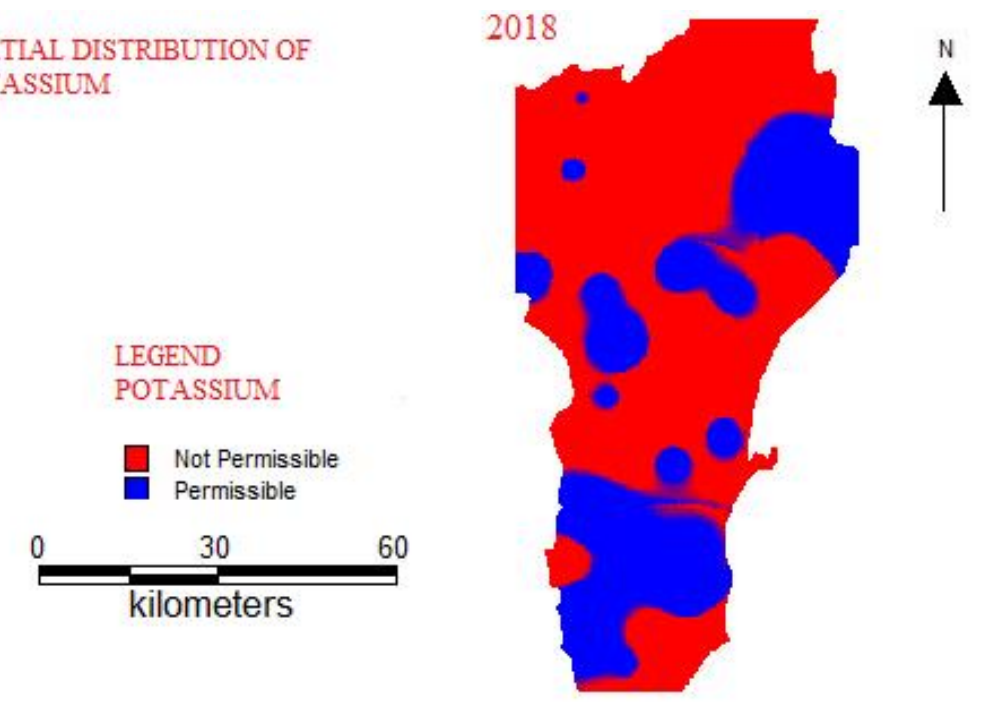

Figure 11. Spatial distribution of potassium 


\section{Bicarbonate}

Bicarbonate ranges from 54 to $702 \mathrm{mg} / \mathrm{l}$ with a mean value of $311.63 \mathrm{mg} / \mathrm{L}$ during the year 2014 and 35 to $732 \mathrm{mg} / \mathrm{L}$ with a mean value of $378.93 \mathrm{mg} / \mathrm{L}$ during the year 2018. Bicarbonate concentrations are somewhat higher in the year 2018 compared to the year 2014 indicating the influence of carbonate due to weathering processes. There is a major variation observed in the spatial distribution of bicarbonate as shown in Figure 12 which may be due to saltwater intrusion (Selvam et al., 2013; Srinivasamoorthy et al., 2011).
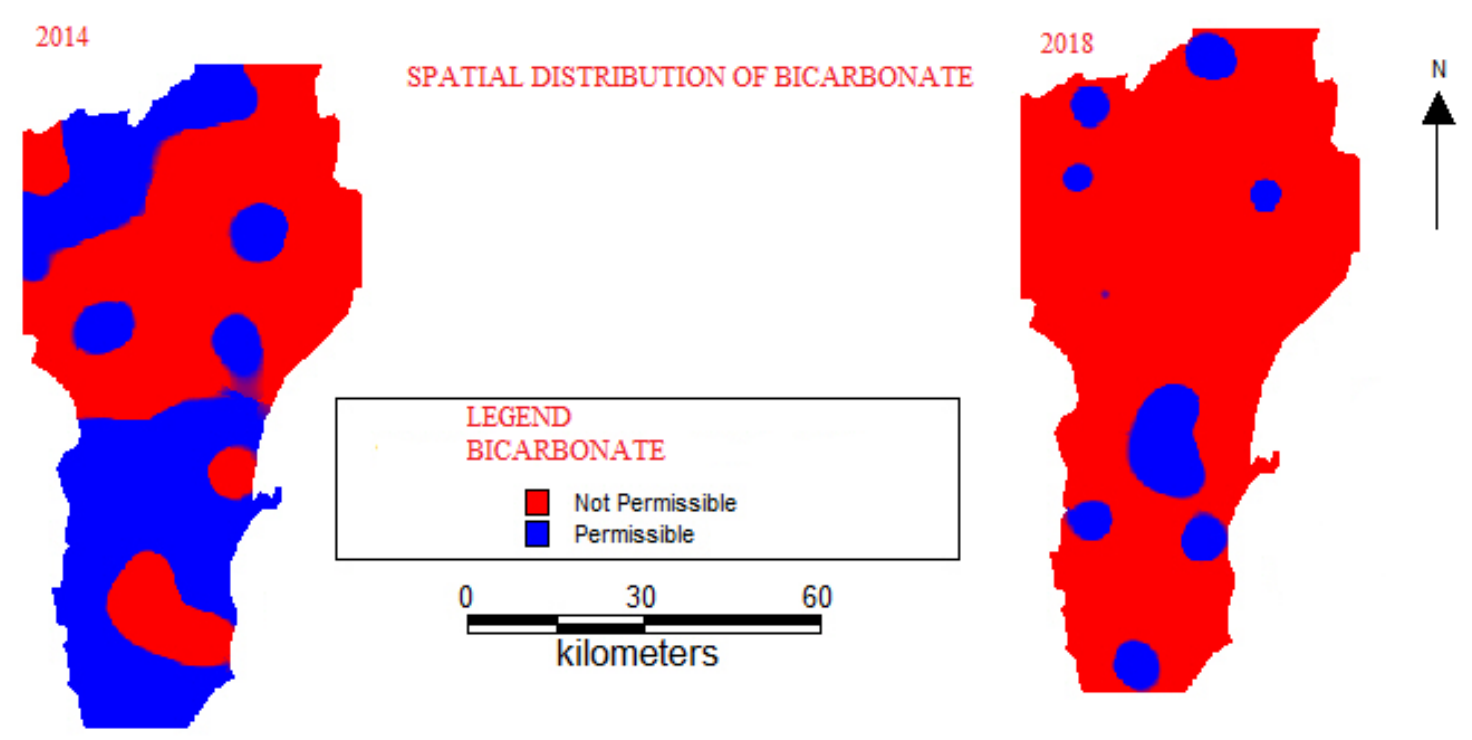

Figure 12. Spatial distribution of bicarbonate

\section{Chloride}

Chloride ranges from 7 to $865 \mathrm{mg} / \mathrm{L}$ with a mean value of $253.03 \mathrm{mg} / \mathrm{L}$ during the year 2014 and 7 to $1914 \mathrm{mg} / \mathrm{L}$ with a mean value of $328.34 \mathrm{mg} / \mathrm{L}$ during the year 2018 . The allowable limit of Chloride is $600 \mathrm{mg} / \mathrm{L}$. In Tuticorin $11 \%$ of sample exceed the permissible limit of chloride concentration in 2014. But in 2018 the percentage increases to $13 \%$. Thus, high concentration of sodium and Chloride ions in groundwater may show a substantial effect of saltwater intrusion (Mondal et al., 2010). The Spatial Distribution of Chloride is shown in Figure 13. Higher concentration is noted during 2018 when compared with 2014 indicating domination of industrial activity and saltwater intrusion to the groundwater. High concentration of Chloride may be harmful to persons suffering from sicknesses of the heart and kidneys (Bhawan and Nagar, 2008).

\section{Sulphate}

Sulphate concentration vary from 10 to $624 \mathrm{mg} / \mathrm{L}$ with a mean value of $145.63 \mathrm{mg} / \mathrm{L}$ during the year 2014 and 6 to $1440 \mathrm{mg} / \mathrm{L}$ with a mean value of $194.08 \mathrm{mg} / \mathrm{L}$ during the year 2018. If the sulphate concentration exceeds allowable limit of $400 \mathrm{mg} / \mathrm{L}$ it will affect human organs. The sorting of groundwater based on sulphate is specified in Table 2 and it is found that $5 \%$ of samples exceeded permissible limits during the year 
2014 and $13 \%$ of samples are found to be exceeded the limits during the year 2018 . Sulphate concentration in groundwater increases due to dissolution minerals and anthropogenic sources. The Spatial Distribution of Sulphate is shown in Figure 14.
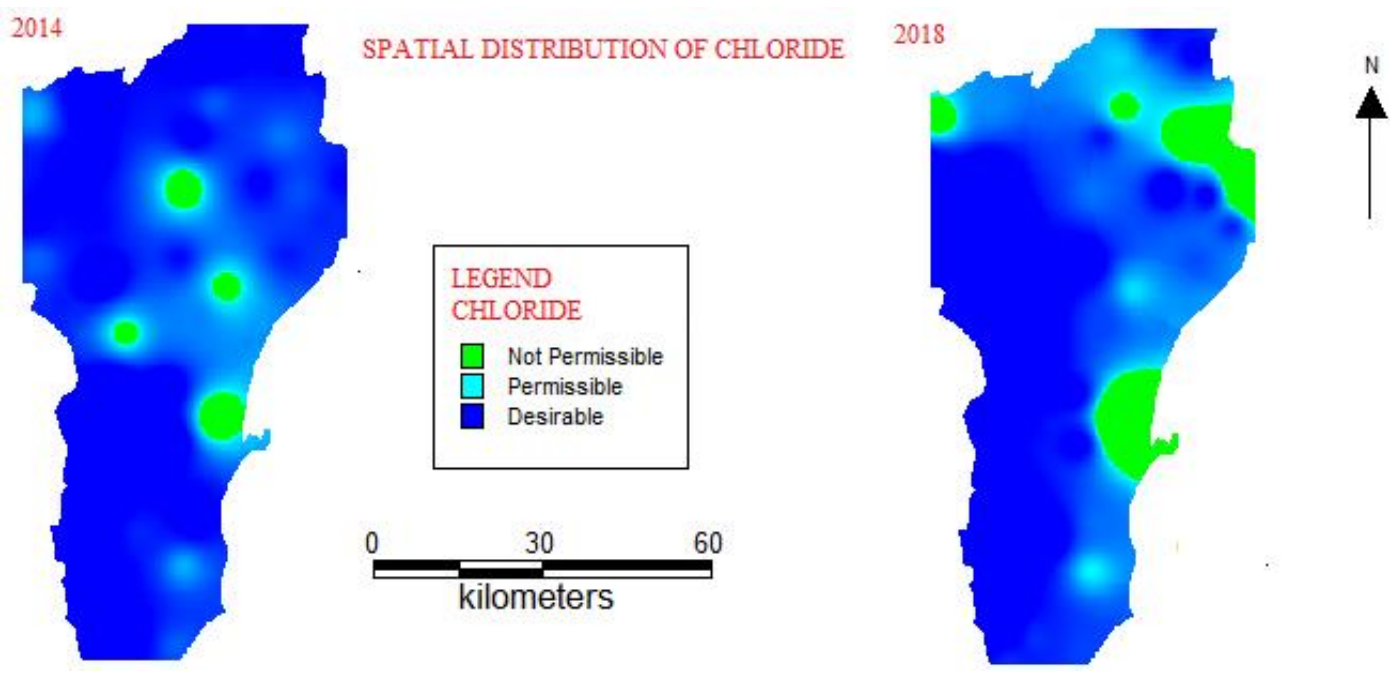

Figure 13. Spatial distribution of chloride
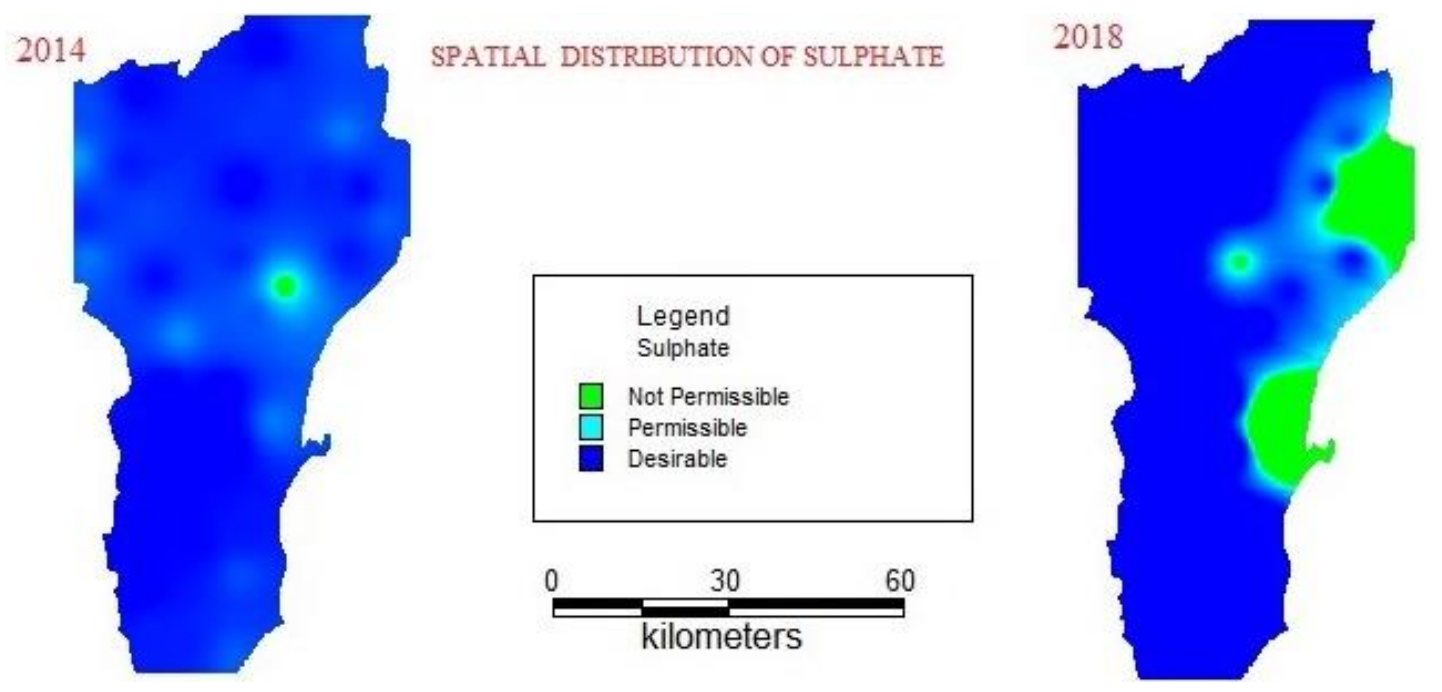

Figure 14. Spatial distribution of sulphate

\section{Nitrate}

Nitrate concentration vary from 1 to $73 \mathrm{mg} / \mathrm{L}$ with a mean value of $45.55 \mathrm{mg} / \mathrm{L}$ during the year 2014 and 1 to $55 \mathrm{mg} / \mathrm{L}$ with a mean value of $43.55 \mathrm{mg} / \mathrm{L}$ during the year 2018 allowable nitrate in drinking water as per WHO standards are $45 \mathrm{mg} / \mathrm{L}$. The spatial distribution of nitrate is shown in Figure 15.

\section{Fluoride}

Fluoride concentration varies from 0 to $2 \mathrm{mg} / \mathrm{L}$ with a mean value of $0.64 \mathrm{mg} / \mathrm{L}$ during the year 2014 and 0 to $1 \mathrm{mg} / \mathrm{L}$ with a mean value of $0.46 \mathrm{mg} / \mathrm{L}$ during the year 
2018. Allowable fluorides in drinking water as per WHO standards are $1.5 \mathrm{mg} / \mathrm{L}$. Concentrations higher than allowable limit create many health problems (WHO, 2017). The spatial distribution of fluoride is shown in Figure 16.
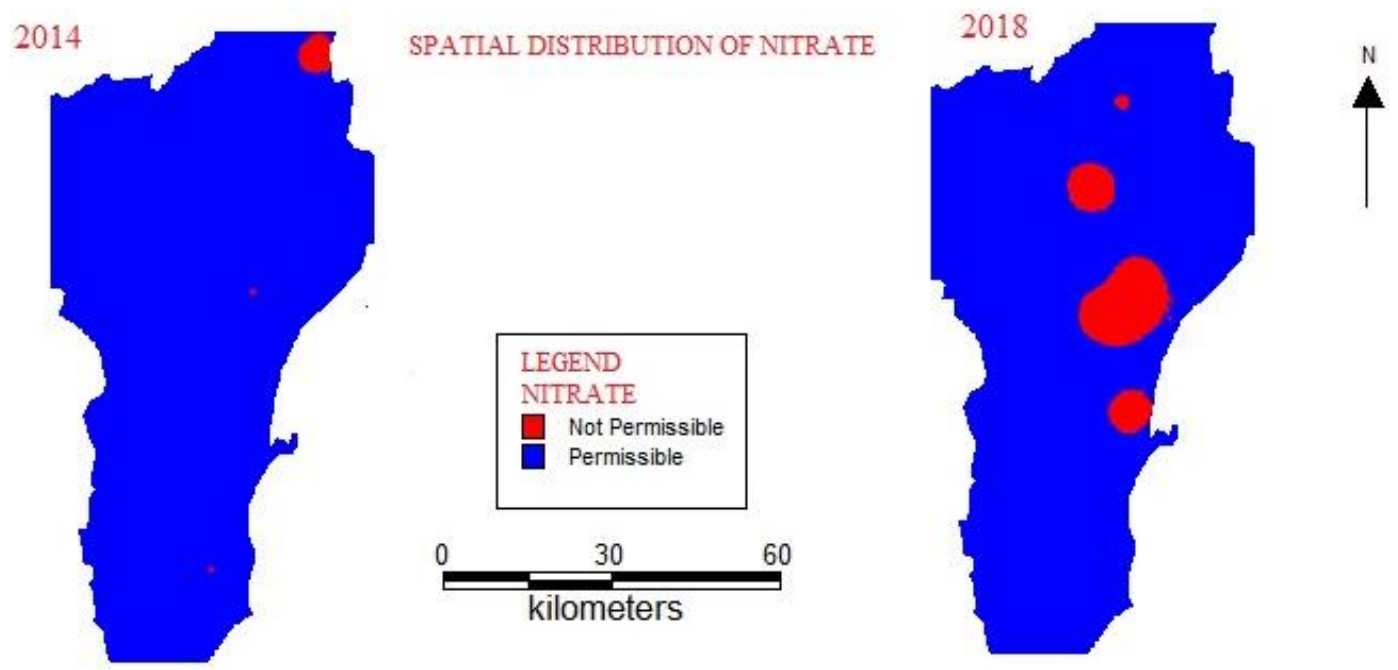

Figure 15. Spatial distribution of nitrate
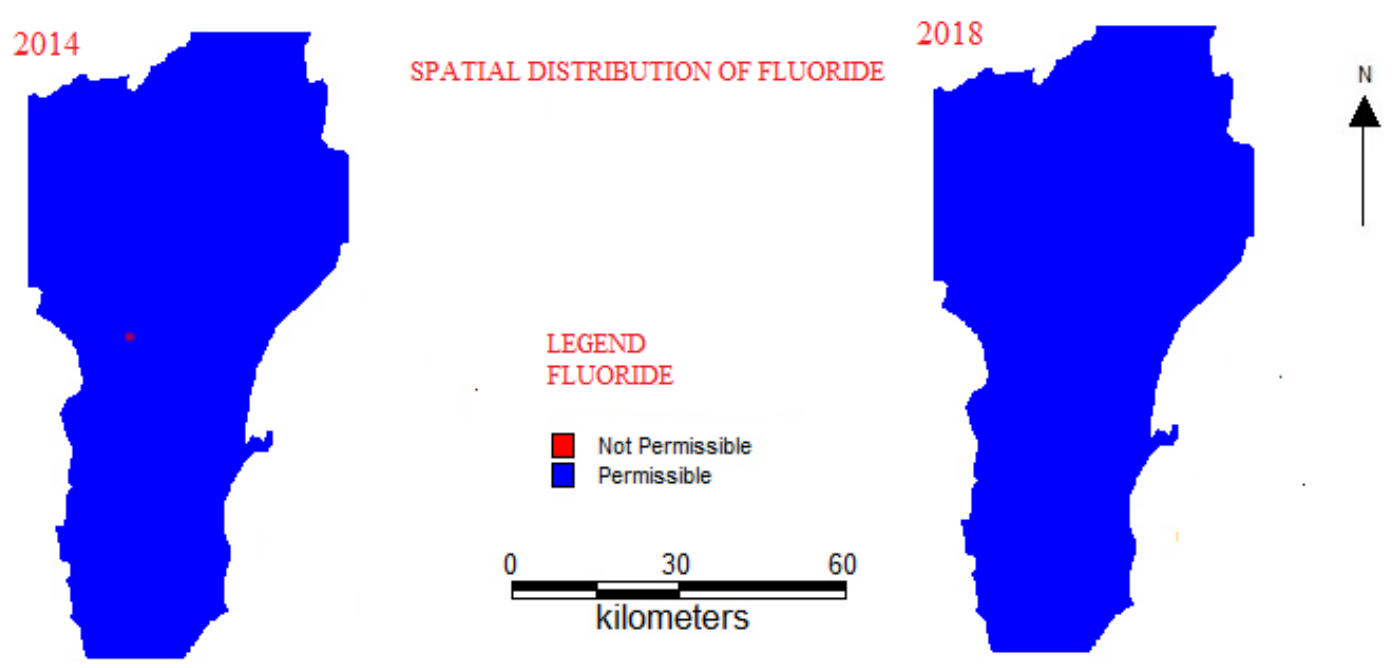

Figure 16. Spatial distribution of fluoride

According to salt concentration cations are characterized in ascending order as follows $\mathrm{Na}^{+}>\mathrm{K}^{+}>\mathrm{Mg}^{2+}>\mathrm{Ca}^{2+}$. From the above sorting it is clearly understood that Sodium and potassium concentration is prominent than the magnesium and calcium.

According to salt concentration anion are characterized in ascending order as follows $\mathrm{HCO}^{3-}>\mathrm{Cl}^{-}>\mathrm{SO}_{4}{ }^{2-}>\mathrm{F}$. From the above sorting, $\mathrm{Cl}$ and $\mathrm{HCO}_{3}$ showed a prominent role in Tuticorin than the concentrations of $\mathrm{NO}_{3}$ and $\mathrm{F}$.

\section{Piper plot}

Statistical diagram such as Piper plot is used to understand about hydro chemical processes functioning in the groundwater. The Piper diagram is used to recognize 
problems concerning the hydrogeochemical development of groundwater. The piper plot has three portions like two triangular portion and a diamond-shaped portion (Piper, 1944). The overall water quality is represented in the diamond-shaped field by extending the position of the plots in the triangular portion. Groundwater can be notable by their location, occupying certain spaces of the diamond-shaped portion. The experimental data obtained from the groundwater samples are plotted on a Piper trilinear diagram to understand the hydrogeochemical pattern in Tuticorin during the years 2014 and 2018.

The geochemical characteristics can be studied using Piper plot, which is separated into six sub groups (1) (Ca-HCO3 type); (2) (Na-Cl type); (3) (mixed Ca-Na-HCO3 type); (4) (mixed Ca-Mg-Cl type); (5) (Ca-Cl type) and (6) (Na-HCO3 type). The diagram Figure 17 exposed that the samples during 2014 found in the group of $\mathrm{Na}-\mathrm{Cl}$ and mixed $\mathrm{Ca}-\mathrm{Mg}-\mathrm{Cl}$ type. But during 2018, Ca-HCO3 type of water prevails in Tuticorin. Ca-Mg-Cl type of water falls under both years. It is detected that the alkalis $\left(\mathrm{Na}^{+}\right.$and $\left.\mathrm{K}^{+}\right)$exceed the alkaline $\left(\mathrm{Ca}^{2+}\right.$ and $\left.\mathrm{Mg}^{2+}\right)$ and strong acids exceed weak acids. During 2014 and 2018, strong acid dominant over weak acid and $\mathrm{HCO}^{3-}$ and $\mathrm{Cl}^{-}$have influence equal to $\mathrm{Na}^{+}$, which indicates the severe saltwater intrusion occurred in Tuticorin.
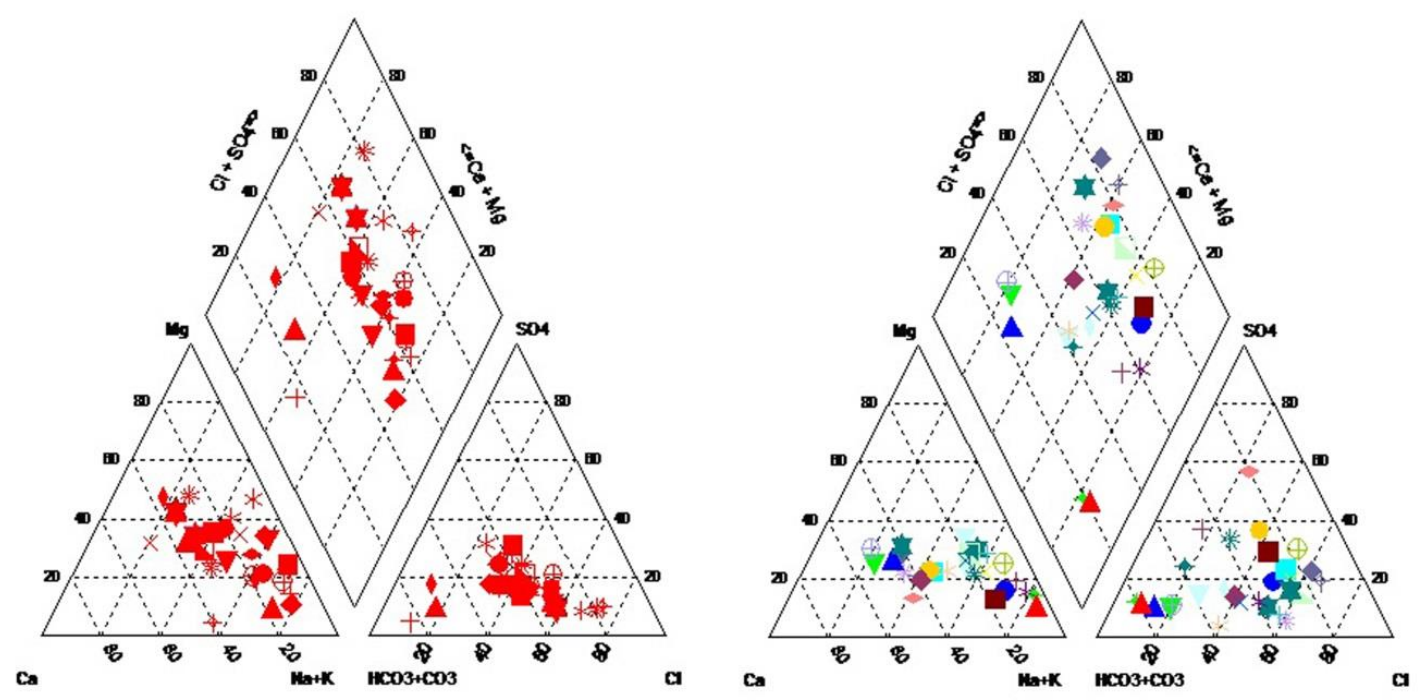

Figure 17. Piper plot during the years 2014 and 2018

\section{Water quality index}

The WQI for groundwater samples is exposed in Tables 6 and 7. Water collected from 38 observation wells are analysed for WQI. From the WQI value, it is found that villages like Keelairal, Kulathurterkku, Ottapidaram, Padanthapuli, Puthiyamputhur, Vilathikulam, Singathakuruchi, Vedanatham, Kachanavilai, Palangulam, Ariyanayagipuram, Srivaikundam, Thirukalur have showed improved water quality in 2018 compared with 2014. Water quality improvement may be due to the less withdrawal fresh groundwater and the rehabilitation of tanks. These villages located in the Taluk of Vilathikulam, Kovilpatti, Satankulam, Ettayapuram, Ottapidaram and Srivaikundam. The sample collected in villages present in Tuticorin Taluk like Melathattaparai, Marthadampatti have showed severe water quality deterioration 
occurred from 2014 to 2018. Since the fresh groundwater extraction is more in Tuticorin due to industries saltwater intrusion occurs in the coastal aquifer. It is clearly portrayed from WQI method that the saltwater intrusion mainly in the coastal belt of Tuticorin and moves inward.

The spatial and attribute data are integrated for the creation of the spatial WQI map. The WQI Maps for the year 2014 and 2018 are depicted in Figure 18.

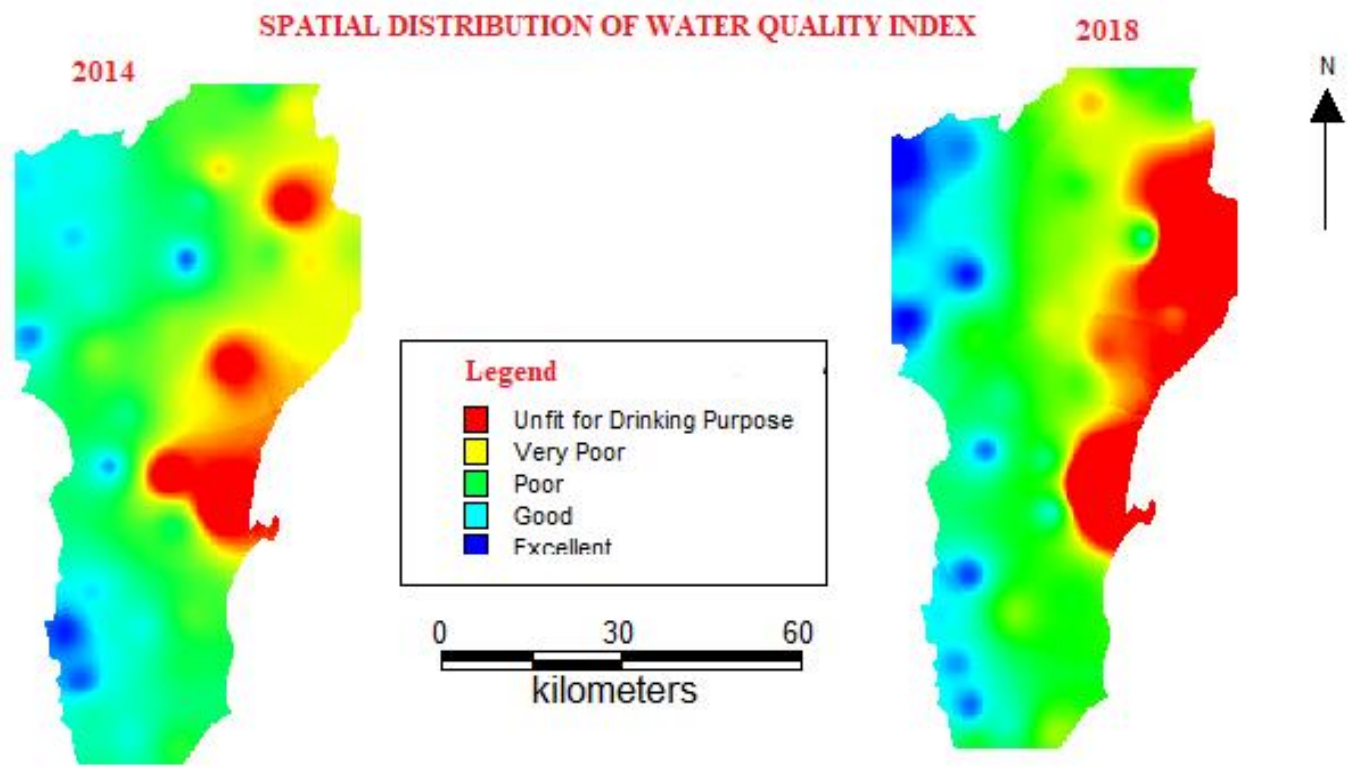

Figure 18. Water quality index map for the year 2014 and 2018

\section{Comparison of WQI with the distance from sea}

The computed WQI with respect to the distance of observation well from the sea is shown in Figure 19. From the figure it can be seen that water quality is poor in the observation wells found with in $15 \mathrm{~km}$ from sea shore during the year 2018 where as in 2014, observation wells found within $9 \mathrm{~km}$ are only affected. Similarly, in 2018 observation wells found with in $7 \mathrm{~km}$ is unfit for drinking. Hence the saltwater intrusion has moved inward from the seashore to the distance of $15 \mathrm{~km}$ during 2018.

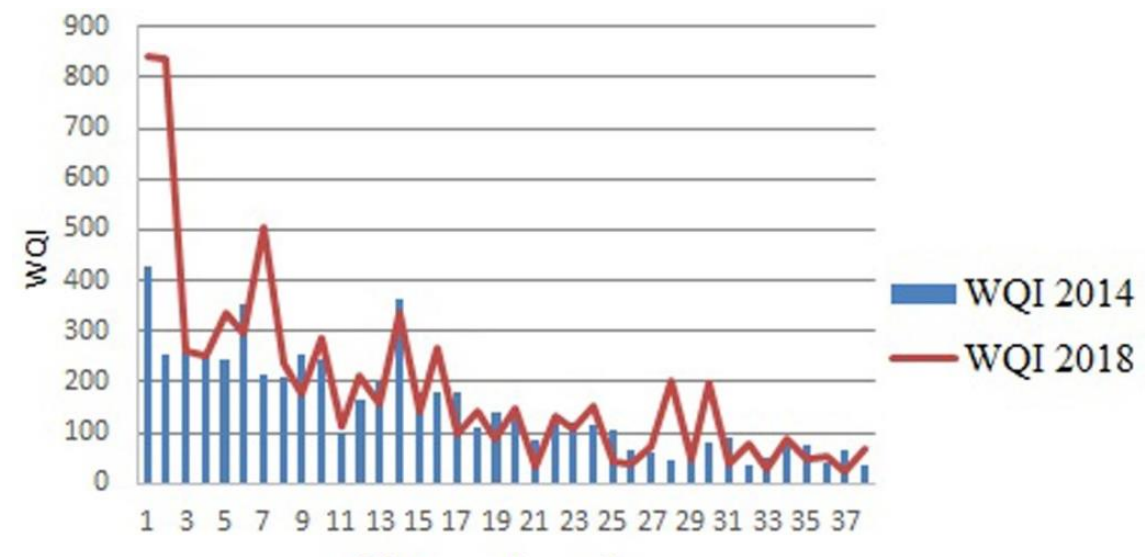

Distance from Sea

Figure 19. WQI with respect to the distance from sea 


\section{Model calibration}

The model is calibrated for steady state and then for transient state. In a steady state calibration hydraulic conductivity and the recharge concentration are adjusted until hydraulic head measured using piezometer in 15 observation wells in April 2018 become close to the computed hydraulic heads. The calibrated results are depicted in Figure 20. Good match is found between observed and computed hydraulic head. For transient state calibration longitudinal and transverse dispersivity are changed to match observed salt concentration with computed salt concentration. Final calibrated value of longitude dispersivity is $30 \mathrm{~m}$ and transverse dispersivity is $4 \mathrm{~m}$.

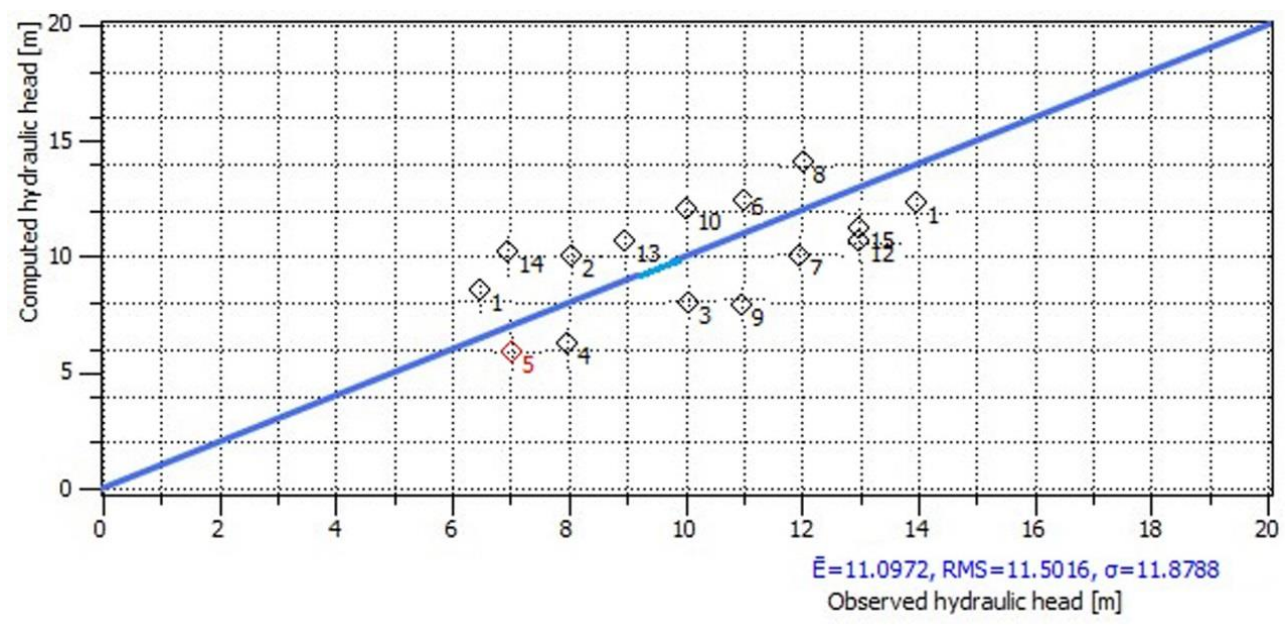

Figure 20. Comparison of measured and computed hydraulic head

\section{Model verification}

The Model developed is validated during the years 2000 to 2018 for the well located in Tuticorin by using data of salt concentration obtained from State Ground and Surface Water Resources Data Centre, Tamilnadu, India. A good match is found between the observed and simulated salt concentration in Tuticorin as shown in Figure 21.

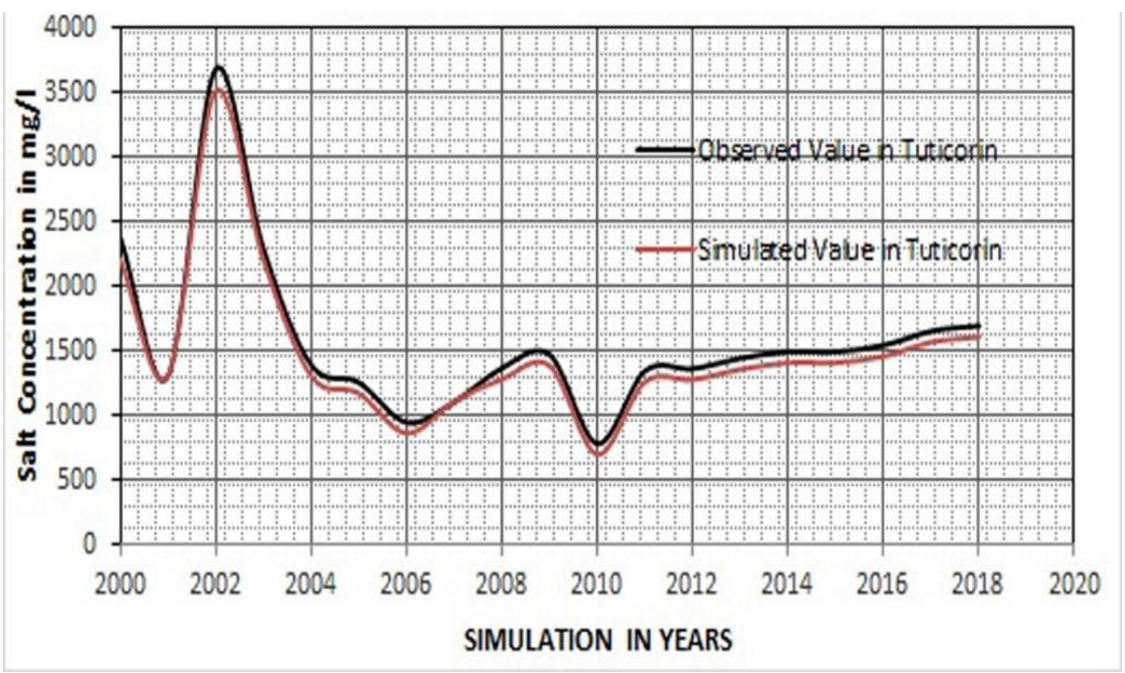

Figure 21. Model verification for the well in Tuticorin 


\section{Model simulation}

The effect of Scenario A (Abstraction) Abstraction of saline groundwater using 15 pumping wells at a withdrawal rate of $1000 \mathrm{~m}^{3} /$ day per well at a short distance of $1500 \mathrm{~m}$ from the shoreline is showed a significant change in salt concentration in Tuticorin as shown in Figure 22.

The effect of Scenario A (Abstraction) Abstraction of saline groundwater using 15 pumping wells at a withdrawal rate of $1000 \mathrm{~m}^{3} /$ day per well at a short distance of $1500 \mathrm{~m}$ from the shoreline is showed a significant change in salt concentration in Tuticorin as shown in Figure 22. Simulated results of Scenario A (Abstraction) in the well in Tuticorin showed a decrease in groundwater salt concentration from $1600 \mathrm{mg} / \mathrm{L}$ in the year 2019 to $900 \mathrm{mg} / \mathrm{L}$ in the year 2030 .

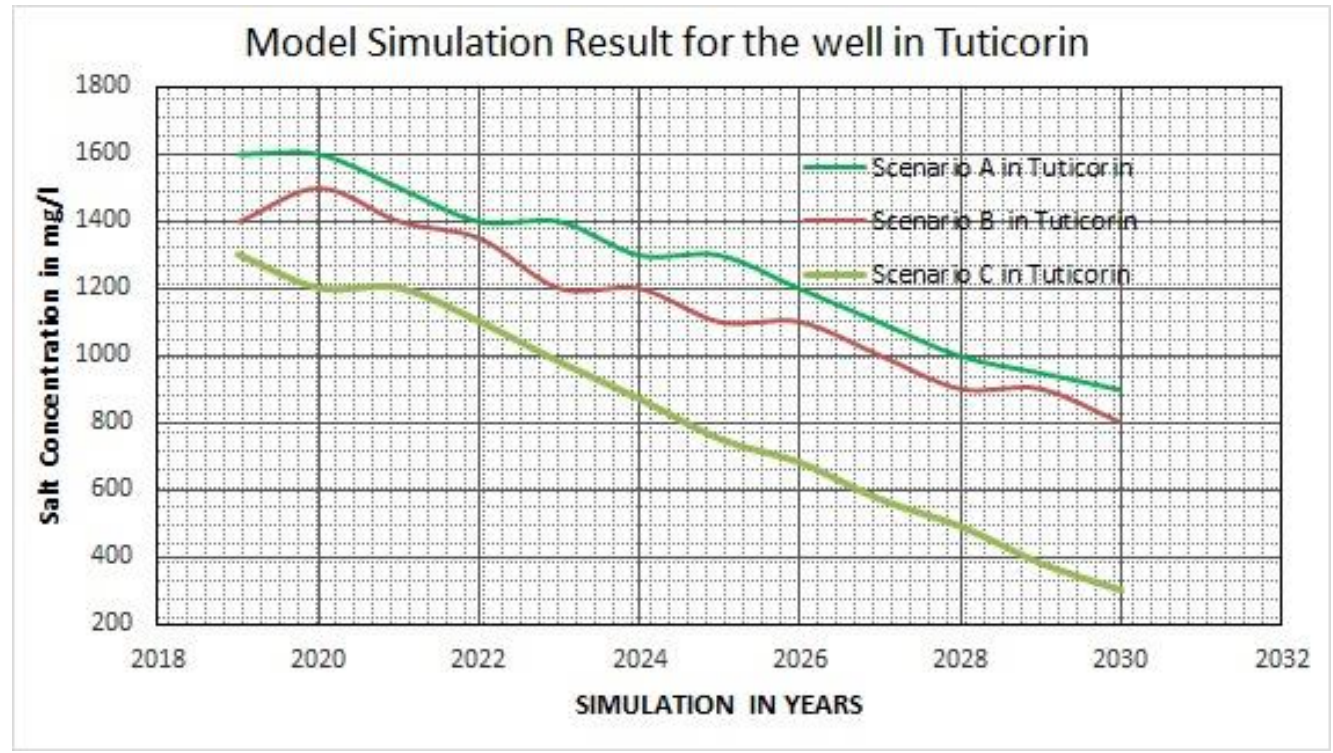

Figure 22. Simulation result for the well in Tuticorin

The Scenario B is the recharge of aquifer with treated saline water at the rate of $500 \mathrm{~m}^{3} /$ day, showed to have significant impact than the pumping Scenario A as depicted in Figure 22. Simulated results of Scenario B (Recharge) in the well in Tuticorin exhibited a decrease in groundwater salt concentration from $1400 \mathrm{mg} / \mathrm{L}$ in the year 2019 to $800 \mathrm{mg} / \mathrm{L}$ in the year 2030 .

But in Scenario C abstracting saline groundwater $1000 \mathrm{~m}^{3} /$ day and recharge treated water at the rate of $500 \mathrm{~m}^{3} /$ day showed good control over the salt water intrusion in Tuticorin as revealed in Figure 22. Simulated results of Scenario C (ADR) in the well in Tuticorin exposed a steady decline in groundwater salt concentration from $1300 \mathrm{mg} / \mathrm{L}$ in the year 2019 to $300 \mathrm{mg} / \mathrm{l}$ in the year 2030 .

From the simulation result it is understood that ADR method is good to control water quality in the well from saltwater intrusion.

\section{Discussion}

From the Spatial analysis of water quality parameters, WQI method, Piper plot diagram, it is understood that the severe saltwater intrusion is occurred in Tuticorin. The 
current pumping rate would further decline the water quality and can lead to the destruction of groundwater resources.

An attempt is made in the study to determine the distance of seawater intrusion based on WQI. The analysis results give better knowledge of zone of dispersion. Water Quality became very poor in the observation wells found with in $15 \mathrm{~km}$ from sea shore in 2018. Hence the saltwater intrusion has moved inward from the seashore to a distance of $15 \mathrm{~km}$. Pumping saline water from the zone of dispersion can reduce saltwater intrusion.

Several studies like Selvam et al. (2013) analysed the water quality parameter but the control measures not provided. In this research groundwater model is developed and three scenarios were applied to find the best method to minimise the seawater intrusion. The simulation results showed that ADR method is good to control from saltwater intrusion.

From the study, it is clearly depicted 15 pumping wells at a withdrawal rate of $1000 \mathrm{~m}^{3} /$ day per well at a short distance of $1500 \mathrm{~m}$ from the shoreline and Recharge treated water at the rate of $500 \mathrm{~m}^{3} /$ day required for the reduction, or displacement of the saline water towards the coast to eventually maintain or push the dispersion zone towards the coast is very important.

\section{Conclusions}

The effect of saltwater intrusion in coastal aquifer necessitates systematic investigation, especially when the extraction of groundwater in the coastal aquifer increases. The spatial analysis of groundwater using GIS, Water Quality Indices method, Piper plot diagram shows that the coastal aquifer is severely affected by saltwater intrusion. Potential intrusion of saltwater is studied with respect to distance of observation wells from seashore. In 2018 water quality in the observation wells located with in $15 \mathrm{~km}$ from the Seashore is very much affected. Three different simulation scenarios namely Abstraction, Recharge and a combination of system were applied to the observation well located in Tuticorin to reduce the seawater intrusion. FEFLOW model was used to evaluate these methods adopted to reduce seawater intrusion. The simulation results of FEFLOW show that the method ADR performs better than using abstraction or recharge wells. Integrating field study with developed model to determine the site selection of recharge well is a possibility for future work.

\section{REFERENCES}

[1] Azeez, P. A., Nadarajan, N. R., Mittal, D. D. (2000): The impact of a monsoonal wetland on groundwater chemistry. - Pollution Research 19(2): 249-255.

[2] Barlow, P. M., Reichard, E. G. (2010): Saltwater intrusion in coastal regions of North America. - Hydrogeology Journal 18(1): 247-260.

[3] Davis, S. N., Dewiest, R. J. M. (1966): Hydrogeology. Vol. 463. - Wiley, New York.

[4] El Mokhtar, M., Chibout, M., El Mansouri, B., Chao, J., Kili, M., El Kanti, S. M. (2018): Modelling of the Groundwater flow and Saltwater Intrusion in the Coastal Aquifer of Fum Al Wad, Province of Laayoan, Morocco. - International Journal of Geo Sciences 9: 71-92.

[5] Freeze, R. A., Cherry, J. A. (1979): Groundwater. - Prentice Hall, Englewood Cliffs.

[6] Gebrehiwot, A. B., Tadesse, N., Jigar, E. (2011): Application of water quality index to assess suitablity of groundwater quality for drinking purposes in Hantebet watershed, 
Tigray, Northern Ethiopia. - ISABB Journal of Food and Agricultural Sciences 1(1): 2230 .

[7] Handa, B. K. (1969): Description and classification of media for hydro-geochemical investigations. - Symposium on Groundwater Studies in Arid and Semiarid Regions, Roorkee.

[8] Hussain, M. S., Javadi, A. A., Ahangar-Asr, A., Farmani, R. (2015): A surrogate model for simulation - optimization of a aquifer systems subjected to seawater intrusion. Journal of Hydrology 523: 542-554.

[9] Idowu, T. E., Ezekiel, N. M., Korowe, M. (2016): Seawater intrusion vulnerability assesment of a coastal aquifer: north coast of Mombasa, Kenya as a case study. International Journal of Engineering Research and Application 6(8): 37-45.

[10] Jebastina, N., Arulraj, G. P. (2017): GIS based assessment of groundwater quality in Coimbatore District, India. - Journal of Environmental and Analytical Toxicology 7(3): $1-9$.

[11] Jebastina, N., Prince Arulraj, G. (2016): Contamination analysis of groundwater in Coimbatore District, India: a statistical approach. - Environmental Earth Sciences 75: 1447.

[12] Kalpana, G. R., Nagarajappa, D. P., Sundar, K. S., Suresh, B. (2014): Determination of groundwater quality index in Vidyanagar, Davanagere City, Karnataka State, India. International Journal of Engineering and Innovative Technology (IJEIT) 3(12): 90-99.

[13] Mondal, N. C., Singh, V. P., Singh, V. S., Saxena, V. K. (2010): Determining the interaction between groundwater and saline water through groundwater major ions chemistry. - Journal of Hydrology 388(1-2): 100-111.

[14] Piper, A. M. (1944): A graphic procedure in the geochemical interpretation of water-analyses. - EOS, Transactions American Geophysical Union 25(6): 914-928.

[15] Ramesh, S., Sukumaran, N., Murugesan, A. G., Rajan, M. P. (2010): An innovative approach of drinking water quality index-A case study from Southern Tamil Nadu, India. - Ecological Indicators 10(4): 857-868.

[16] Rao, S. V. N., Sreenivasulu, V., Bhallamudi, S. M., Thandaveswara, B. S., Sudheer, K. P. (2004): Planning groundwater development in coastal aquifers. - Hydrological Sciences Journal 49(1): 155-170.

[17] Sawyer, C., McCarthy, P. (1967): Chemistry for Sanitary Engineering. - McGraw-Hill, New York.

[18] Schoeller, H. (1965): Qualitative Evaluation of Groundwater Resources. Methods and Techniques of Groundwater Investigations and Development. - UNESCO 5483, Paris.

[19] Selvam, S., Manimaran, G., Sivasubramanian, P. (2013): Hydrochemical characteristics and GIS-based assessment of groundwater quality in the coastal aquifers of Tuticorin corporation, Tamilnadu, India. - Applied Water Science 3(1): 145-159.

[20] Sener, E., Sener, S., Davraz, A. (2009): Assessment of aquifer vulnerability based on GIS and DRASTIC methods: a case study of the Senirkent-Uluborlu Basin (Isparta, Turkey). - Hydrogeology Journal 17(8): 2023.

[21] Serrekawo, N., Karuppannan, S. (2018): Groundwater quality assessment using water quality index in Modjo River Basin, Central Ethiopia. - Journal of African Earth Sciences 147: 300-311.

[22] Sherif, M., Kacimov, A. (2008): Pumping of brackish and saline water in coastal aquifers: an effective tool for alleviation of seawater intrusion. - Proceeding of 20th SWIM, Naples, Florida, USA.

[23] Sherif, M. M., Hamza, K. I. (2001): Mitigation of seawater intrusion by pumping brackish water. - Transport in Porous Media 43(1): 29-44.

[24] Song, T., Kim, K. (2009): Development of a water quality loading index based on water quality modeling. - Journal of Environmental Management 90(3): 1534-1543. 
[25] Srinivas, Y., Oliver, D. H., Raj, A. S., Chandrasekar, N. (2013): Evaluation of groundwater quality in and around Nagercoil town, Tamilnadu, India: an integrated geochemical and GIS approach. - Applied Water Science 3(3): 631-651.

[26] Srinivasamoorthy, K., Nanthakumar, C., Vasanthavigar, M., Vijayaraghavan, K., Rajivgandhi, R., Chidambaram, S., Anandhan, P., Manivannan, R., Vasudevan, S. (2011): Groundwater quality assessment from a hard rock terrain, Salem district of Tamilnadu, India. - Arabian Journal of Geosciences 4(1-2): 91-102.

[27] Stigter, T. Y., Ribeiro, L., Dill, A. C. (2006): Application of a groundwater quality index as an assessment and communication tool in agro-environmental policies-Two Portuguese case studies. - Journal of Hydrology 327(3-4): 578-591.

[28] Tavassoli, S., Mohammadi, F. (2017): Groundwater quality assessment based on WQI and its vulnerability to saltwater intrusion in a coastal city, Iran. - Journal of Geo Science and Environment Protection 5: 88-98.

[29] Thomas, A., Eldho, T. I., Rastogi, A. K. (2016): Simulation of seawater intrusion in coastal confined aquifer using a point collocation method based mesh free model. Journal of Water Resource and Protection 8: 534-549.

[30] Tijani, M. N. (1994): Hydrogeochemical assessment of groundwater in Moro area, Kwara State, Nigeria. - Environmental Geology 24(3): 194-202.

[31] Todd, D. K. (1959): Groundwater Hydrology. - John Wiley and Sons, New York.

[32] Todd, D. K. (1974): Salt-Water Intrusion and Its Control. - Journal-American Water Works Association 66(3): 180-187.

[33] Todd, D. K. (1980): Groundwater Hydrology. Second Edition. - John Wiley and Sons, New York.

[34] World Health Organization (2017): Guidelines for Drinking-Water Quality: First Addendum to the Fourth Edition. - WHO, Geneva. 